\title{
Electrophysiological examination of response-related interference while dual-tasking: is it motoric or attentional?
}

\author{
Kyung Hun Jung ${ }^{1}\left[\right.$ Tim Martin $^{1} \cdot$ Eric Ruthruff $^{2}$
}

Received: 23 May 2019 / Accepted: 19 October 2019 / Published online: 4 February 2020

(C) The Author(s) 2020

\begin{abstract}
The possibility that interference between motor responses contributes to dual-task costs has long been neglected, yet is supported by several recent studies. There are two competing hypotheses regarding this response-related interference. The motor-bottleneck hypothesis asserts that the motor stage of Task 1 triggers a refractory period that delays the motor stage of Task 2. The response-monitoring hypothesis asserts that monitoring of the Task-1 motor response delays the responseselection stage of Task 2. Both hypotheses predict lengthening of Task-2 response time (RT2) when Task 1 requires motor processing relative to when it does not. However, they assume different loci for the response-related bottleneck, and therefore make different predictions regarding (a) the interaction between Task-1 motor requirement and the Task- 2 difficulty effect as measured by RT2 and (b) the premotoric durations and motoric durations of Task 2 as measured by lateralized readiness potentials (LRPs). To test these predictions, we conducted two experiments manipulating the Task-1 motor requirement (Go vs. NoGo) and Task-2 response-selection difficulty, as well as the stimulus-onset asynchrony (SOA). Task-1 motor processing significantly lengthened RT2, suggesting response-related interference. Importantly, the Task-1 motor response reduced the Task-2 difficulty effect at the short SOA, indicating postponement of the Task-2 motor stage, consistent with the motor-bottleneck hypothesis. Further consistent with the motor-bottleneck hypothesis, the Task-2 LRP indicated a consistent premotoric duration of Task 2 regardless of Task-1 motor requirement. These results are difficult to reconcile with the response-monitoring hypotheses, which places the response-related bottleneck before the response-selection stage of Task 2. The results also have important implications regarding use of locus-of-slack logic in PRP studies.
\end{abstract}

\section{Introduction}

Research on divided attention shows both efficiency and limitation. While visual perception demonstrates successful divided attention under desirable conditions (Shiffrin \& Schneider, 1977), dual-task performance is often subject to stubborn limitations. These limitations arise even when the component tasks are simple (Vince, 1948) and sometimes even after participants receive extensive practice (Ruthruff, Johnston, \& Van Selst, 2001; Strobach \& Schubert, 2017). The overall goal of the present study is to better understand the nature of dual-task interference. In particular, we aim to elucidate the contribution of the motor stage, which

Kyung Hun Jung

kjung2@kennesaw.edu

1 Department of Psychological Science, Kennesaw State University, 1000 Chastain Road, Kennesaw, GA 30144, USA

2 Department of Psychology, University of New Mexico, Albuquerque, USA traditionally has been neglected as a cause of dual-task interference.

To study the mechanisms of dual-task interference, researchers often use the overlapping-task paradigm, which is also known as the psychological refractory period (PRP) paradigm. In this paradigm, participants make a separate response to each stimulus of two tasks ( $\mathrm{S} 1$ and $\mathrm{S} 2$ for Task 1 and Task 2). Researchers manipulate the degree of temporal overlap between the two tasks by varying the interval between the onsets of S1 and S2 (stimulus-onset asynchrony, SOA). A common finding is that the Task-2 performance is impaired as task overlap increases (i.e., as SOA decreases), which is known as the PRP effect (Telford, 1931; Vince, 1948; Welford, 1952).

The PRP effect implies that, at short SOAs, at least one of the three basic stages of Task 2 (perception, response selection, and motor) is slowed or delayed relative to long SOAs. Thus far, the dominant explanation of the PRP effect has been the response-selection bottleneck hypothesis (Pashler, 1984, 1994; Welford, 1952, but see also Tombu \& Jolicœur, 

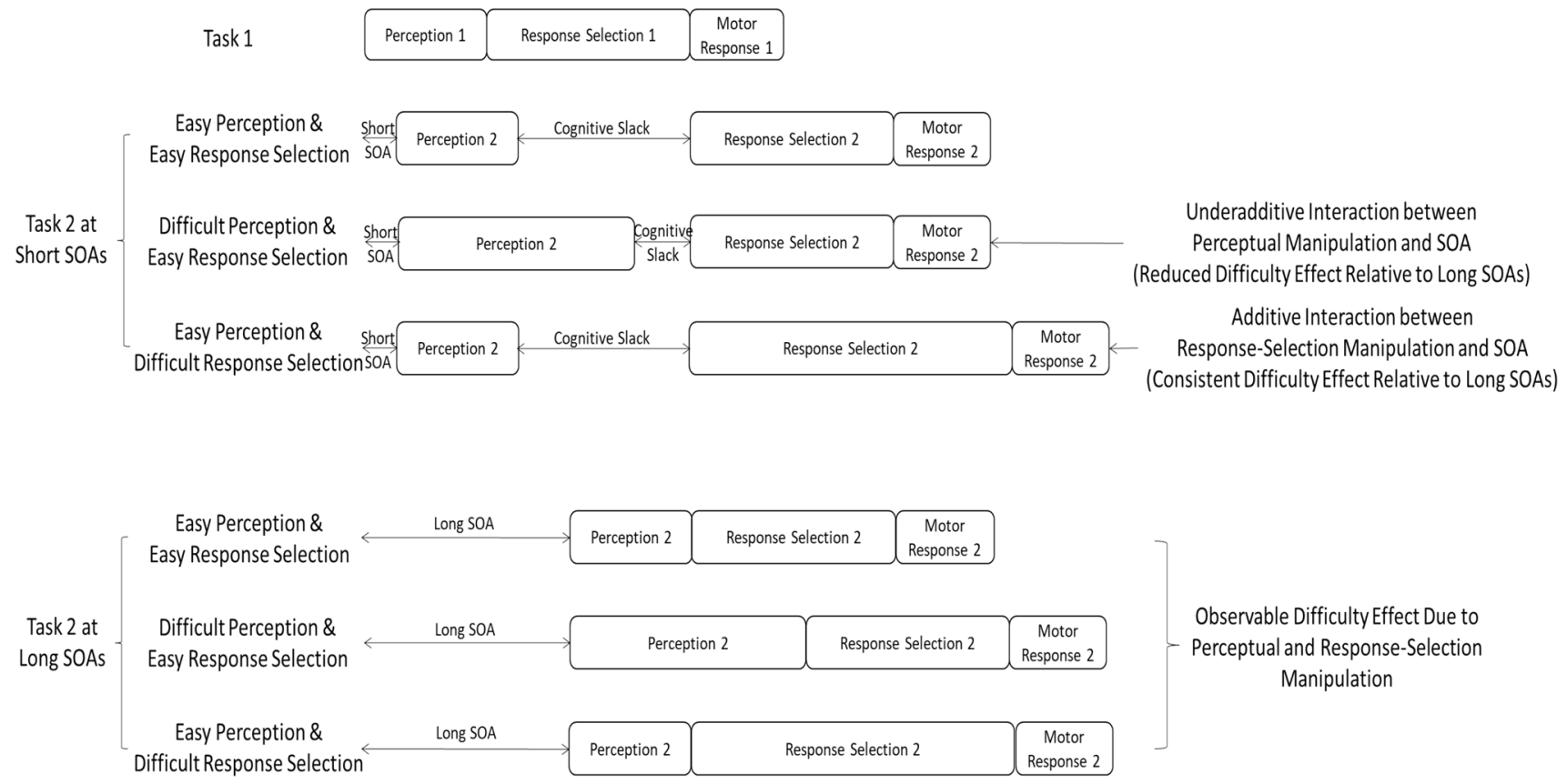

Fig. 1 Cognitive slack hypothesized by response selection bottleneck (RSB) hypothesis. According to RSB hypothesis, A2 manipulation would not be reflected in RT2 (upper panel) while B2 manipulation would (lower panel)

2003, for the capacity-sharing hypothesis), which assumes that only one response-selection stage can access limitedcapacity central attentional resources at a time. According to this hypothesis, while the response-selection stage of Task 1 is accessing the limited resources, Task- 2 response selection cannot operate and must wait until the resources become available. This waiting period (sometimes called cognitive slack) accounts for the PRP effect.

Previous studies have supported the core assumption of the response-selection bottleneck hypothesis-that the major dual-task bottleneck is located at the response-selection stage. For example, using "locus-of-slack" logic (McCann \& Johnston, 1992), Pashler and Johnston (1989) demonstrated that a manipulation of the perceptual difficulty of Task 2 (dim vs. bright S2) caused the expected difficulty effect on response time of Task 2 (RT2) at long SOAs but not at short SOAs (i.e., an underadditive interaction, see Fig. 1). In contrast, a manipulation of the response-selection difficulty of Task 2 (repeated vs. non-repeated responses) showed a consistent difficulty effect on RT2 across SOAs (i.e., an additive interaction). This pattern of interactions is exactly what the response-selection bottleneck hypothesis predicts. Specifically, the effect of manipulations on Task-2 stages prior to the bottleneck (cognitive slack), such as perceptual manipulations, will be absorbed into the period of cognitive slack at short SOAs but not at long SOAs, yielding an underadditive interaction with SOA. Meanwhile, the effect of manipulations of Task-2 stages after the bottleneck, such as response-selection manipulations, cannot be absorbed into the slack, yielding an additive interaction with SOA.

Critical to the present study, the response-selection bottleneck hypothesis assumes that the motor stage does not participate in the dual-task interference (the capacity-sharing hypothesis has the same assumption). That is, the motor stage of a task can operate in parallel with any stage of the other task. Therefore, according to the response-selection bottleneck hypothesis, manipulating the existence or duration of the motor stage of Task 1 would not necessarily affect RT2. However, several studies (see below) have contradicted this prediction, suggesting that the response-selection hypothesis requires some modification.

\section{Response-related interference}

Response-related interference in dual-task performance was first hypothesized based on an underadditive interaction between Task- 2 response-selection difficulty and SOA (in contrast to the additivity observed by Pashler \& Johnston, 1989). Such underadditivity suggests that there is a very late bottleneck (i.e., after the response-selection stage), into which the effect of response-selection manipulation can be absorbed. For example, Karlin and Kestenbaum (1968) employed simple-detection vs. two-choice responses to manipulate the response-selection difficulty of Task 2 and found that the effect of this manipulation diminished at 
short SOAs relative to long SOAs (underadditivity). Based on this finding, Keele (1973) proposed that the initiation of the Task-1 motor response is followed by a motoric refractory period that temporarily prevents initiation of a motor response to Task 2, creating a response-related bottleneck located after the response-selection stage but before the motor stage of Task 2, into which the difficulty effect can be absorbed. Keele further concluded that the primary source of the PRP effect is competition between two motor stages rather than competition between response-selection stages.

De Jong (1993) tested Keele's (1973) proposal by directly manipulating the need for a motor response to Task 1; he used the go-no-go Task 1, which did not require a motor response on half of the trials. He manipulated the responseselection difficulty of Task 2 using the simple-detection vs. two-choice responses, following Karlin and Kestenbaum (1968). The results supported Keele's claim of responserelated interference by showing underadditivity between Task-2 response-selection difficulty and SOA, which was stronger when Task 1 required a motor response (Go trials) than when it did not (NoGo trials). Therefore, De Jong concluded that the Task-1 motor response imposes a bottleneck (in addition to, but separate from, the response-selection bottleneck) between the response-selection and motor stage of Task 2, into which the effect of response-selection difficulty was absorbed.

The underadditive interaction between Task-2 responseselection difficulty and SOA found in Karlin and Kestenbaum (1968) and De Jong (1993) directly contradicts the additive interaction found in Pashler and Johnston (1989). To explain this inconsistency, later researchers pointed to a possible flaw in the way that Karlin and Kestenbaum, as well as De Jong, manipulated Task-2 response-selection difficulty (i.e., simple-detection vs. two-choice responses). For example, Schubert (1999) pointed out that when participants perform a simple-detection task and the SOA is long, they can anticipate the upcoming stimulus and become increasingly prepared to make the predetermined response while waiting for the stimulus. Therefore, the easy condition (simple detection) at long SOAs is even easier than it is at short SOAs. Because such a preparation is not possible in the difficult condition (two-choice responses), the net result would be an artifactual underadditivity between the response-selection difficulty and SOA. In the present study, therefore, we avoided this specific procedure to manipulate Task-2 response-selection difficulty.

Although the above studies comparing simple-detection with two-choice responses were not definitive, other behavioral and physiological evidence support the responserelated interference. For example, Ulrich et al. (2006) manipulated the duration of the Task-1 motor response using a response lever that moved along either a short or long track. They found that RT2 was significantly affected by Task-1 motor-response duration: as the duration increased (tracing a long track relative to a short one), RT2 further increased (from 653 to $736 \mathrm{~ms}$ ) and this increase was even greater at short SOAs than at long SOAs. The results supported the response-related interference assuming that the long-duration motor responses of Task 1 caused greater response-related interference than the short-duration ones especially at short SOAs where Task-2 motor response follows the Task- 1 motor response with a close temporal proximity.

As mentioned above, response-related interference would be most prominent at short SOAs-where the two motor responses are due at nearly the same time-than at long SOAs, which can be examined using lateralized readiness potentials (LRP; Coles, 1989). The LRP is an evoked response potential generated by primary motor cortex M1 with likely contributing sources from other motor cortical areas (Praamstra, Schmitz, Freund, \& Schnitzler, 1999; Sammer et al., 2005). When a participant is preparing to initiate a motor response, M1 in the contralateral hemisphere of the intended hand generates greater currents than M1 in the ipsilateral hemisphere. This discrepancy in the motor cortex activity between the two hemispheres is the source of the LRP. The LRP is believed to occur as early as during the response-selection stage but before the actual motor response, and therefore, can serve as an index of when the motor response is prepared and initiated (Coles, 1989; Miller \& Ulrich, 1998, Miller \& Hackley, 1992). If the LRP is calculated relative to the stimulus onset (S-LRP), it reflects the duration of premotoric stages (perception and response selection, Osman \& Moore, 1993). If the LRP is calculated relative to the response onset (LRP-R), then it reflects the duration of the motor stage. Importantly, while the responseselection bottleneck hypothesis predicts a lengthening of the premotoric stages of Task 2 only (as measured by S2-LRP) as SOA decreases, if the duration of the motor stage (as measured by LRP-R2) increases as SOA decreases, then, it would be more compatible with the notion of responserelated interference. Sommer, Leuthold, and Schubert (2001) replicated Karlin and Kestenbaum (1968)—with the manipulation of whether Task 2 required simple-detection or two-choice responses-while measuring LRPs. In the simple-detection condition, they observed LRP evidence that participants did in fact initiate the response early, in anticipation of the stimulus, consistent with Schubert's (1999) anticipation account. Therefore, they concluded that the earlier evidence of response-related bottleneck that involved a simple-detection condition was due to the increased anticipation of S2 in the simple-detection condition at long SOAs rather than the response-related bottleneck itself. However, in the two-choice-response condition, which is the typical experimental setting of the PRP paradigm, they found that as SOA decreased (from 700 to $100 \mathrm{~ms}$ ), both the premotoric 
duration as measured by the S-LRP and the motor-response duration as measured by the LRP-R of Task 2 increased: $245-\mathrm{ms}$ increase in the premotoric and 55-ms increase in the motoric duration. Such an increase of motoric duration of Task 2 at short SOAs relative to long SOAs is consistent with the response-related interference.

In sum, the notion of response-related interference was originally proposed based on the underadditive interaction between Task-2 response-selection difficulty and SOA, which was later criticized as a possible artifact of using simple detection. However, response-related interference has also been supported by an effect of the Task- 1 motorstage duration on RT2 (Ulrich et al., 2006) as well as by lengthening of the Task-2 motor-stage duration at short SOAs as measured by LRPs (Sommer et al., 2001) —in both studies, the effect of response-related interference was less than $100 \mathrm{~ms}$. Below, we address two competing hypotheses regarding the nature of the response-related interference. Although both hypotheses assert that Task-1 motor stage causes a processing bottleneck in Task 2 , they assume the bottleneck in different locations of Task 2 .

\section{Two hypotheses regarding the nature of the response-related interference}

Regarding the nature and locus of the response-related interference, there are two prominent competing views: the motor-bottleneck hypothesis and the response-monitoring hypothesis. The motor-bottleneck hypothesis suggests that the nature of response-related interference is purely motoric. Specifically, it assumes that the initiation of the Task-1 motor response is followed by a motoric refractory period that temporarily prevents the initiation of the Task- 2 motor response (Keele, 1973). Therefore, according to this hypothesis, the Task-1 motor response generates an additional slack after the response-selection but before the motor stage of Task 2 . A competing view, the response-monitoring hypothesis, suggests that the nature of the response-related interference is attentional rather than motoric. This hypothesis is based on Welford's (1952) assumption that a motor response is accompanied by a monitoring process that consumes the same capacity-limited central attentional resources as response selection. Therefore, according to the response monitoring hypothesis, Task- 2 response selection does not merely wait for completion of Task-1 response selection but also for completion of the Task-1 response and the monitoring of that response. In other words, Task-1 response monitoring adds a period of slack before the response-selection stage of Task 2, in a sense extending the cognitive slack already created by the response-selection bottleneck.

Before describing evidence supporting each of the two hypotheses, we should emphasize the critical distinction between the two hypotheses: whether the additional processing bottleneck in Task 2 triggered by the Task- 1 motor response is located before or after the response-selection stage of Task 2, which could affect RT2 and Task-2 LRP differentially. Regarding RT2, because the motor-bottleneck hypothesis assumes the response-related bottleneck after the response-selection stage of Task 2 (noted as additional slack in a in Fig. 2), it allows absorption of any Task-2 response-selection-difficulty effects into the slack, resulting in an underadditive interaction between response-selection difficulty and SOA. In contrast, because the response-monitoring hypothesis assumes the additional slack before the response-selection stage of Task 2 (b in Fig. 2), the effect of Task-2 response-selection difficulty cannot be absorbed into the slack, resulting in an additive interaction between response-selection difficulty and SOA on RT2. Regarding the LRP, the motor-bottleneck hypothesis suggests that the Task-1 motor response lengthens the duration of Task 2 after the response-selection stage (because the additional slack is inserted after the response selection of Task 2); in contrast, the response-monitoring hypothesis suggests that the Task-1 motor response lengthens the duration before response selection (because the additional slack is inserted before the Task-2 response selection).

\section{Evidence for the motor-bottleneck hypothesis}

Bratzke, Rolke, and Ulrich (2009) manipulated the duration of the Task-1 motor response (tracing a short or long track) as well as the difficulty of Task-2 response selection (compatible vs. non-compatible stimulus-response mapping). They found that the Task-2 difficulty effect was smaller at short SOAs than at long SOAs-121 ms vs. $165 \mathrm{~ms}$ (underadditivity) — and this underadditivity was more prominent when the Task-1 motor-response duration was long (67-ms interaction) than when it was short (22-ms interaction). This is just the result one would expect from the motor-bottleneck hypothesis: lengthening of the Task-1 motor response further lengthens the additional slack period before the Task- 2 motor response, into which the effect of Task-2 response-selection difficulty can be absorbed. Meanwhile, the response-monitoring hypothesis, which assumes additional slack before the response-selection stage of Task 2 , incorrectly predicts an additive interaction between Task- 2 response-selection difficulty and SOA.

\section{Evidence for the response-monitoring hypothesis}

Evidence supporting the response-monitoring hypothesis involves what is known as the residual PRP effect: even without any temporal overlap between the processes of Task 1 and Task 2-because $\mathrm{S} 2$ is presented after the completion of Task $1-\mathrm{RT} 2$ is still prolonged at short SOAs relative to long SOAs. As an example of such a residual PRP effect, 


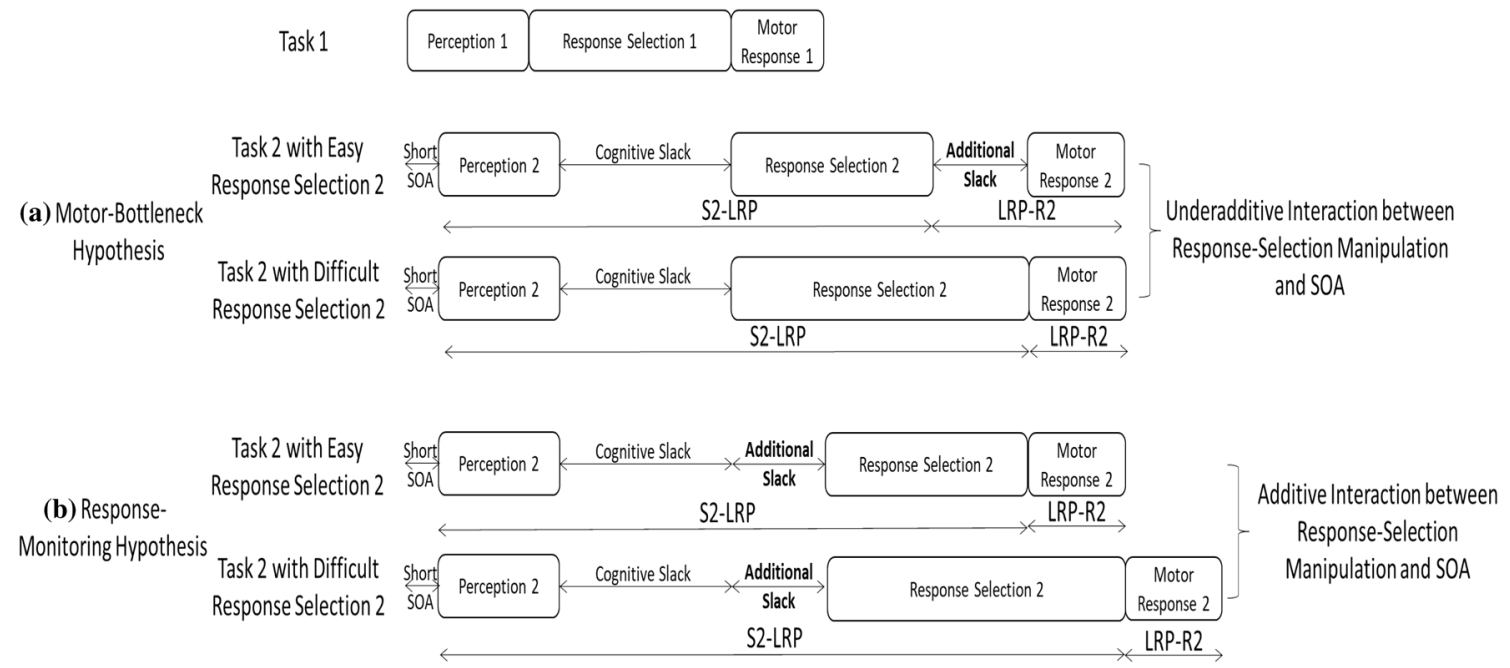

Fig. 2 Example processing time diagrams illustrating the distinct predictions of the motor-bottleneck and response-monitoring hypotheses (i.e., where the additional response-related bottleneck occurs). a The motor-bottleneck hypothesis assumes that the Task-2 motor stage is delayed by the Task-1 motor stage, resulting in a response-related bottleneck (denoted as 'additional slack') located after Task-2 response selection. b Meanwhile, the response-monitoring hypothesis assumes that Task-2 response selection is delayed while monitoring the Task-1 responses, resulting in a response-related bottleneck before the response-selection stage of Task 2 . Therefore, the motor-bottleneck hypothesis allows underadditivity between Task- 2 response-selection

in one condition of Jentzsch, Leuthold, and Ulrich (2007), mean RT2 was $40 \mathrm{~ms}$ longer at the short SOA (400 ms) than the long SOA (600 ms) even though S2 was presented after the completion of Task 1.

To evaluate the predictions of the motor-bottleneck and response-monitoring hypotheses regarding the residual PRP effect, Jentzsch et al. (2007) analyzed the LRP and found that the premotoric duration of Task 2 (S2-LRP) increased as SOA decreased, while the motor-response duration (LRPR2) was consistent across SOAs (unlike the findings of Sommer et al., 2001). This result, along with their behavioral data, is more compatible with the response-monitoring hypothesis that assumes the additional slack due to Task-1 motor response before the Task-2 response selection than the motor-bottleneck hypothesis.

In sum, behavioral measures (RT2) obtained with a Task-1 motor-duration manipulation supported the motorbottleneck hypothesis by showing prominent underadditivity between Task- 2 response-selection difficulty and SOA especially with a long motor stage of Task 1 (Bratzke et al., 2009). In contrast, the LRP data regarding the residual PRP effect supported the response-monitoring hypothesis by showing a lengthened premotoric duration of Task 2 at short SOAs along with a consistent motor-stage duration across SOAs (Jentzsch et al., 2007; but see also, Sommer et al., 2001). We should note that the evidence supporting each difficulty and stimulus-onset asynchrony (SOA) due to absorption of Task-2 difficulty effect into the additional slack, but the responsemonitoring hypotheses does not. Regarding the LRP, the motor-bottleneck hypothesis assumes that the Task-1 motor response lengthens the duration of Task 2 after the response-selection stage (because the additional slack is inserted after the response selection of Task 2), and this effect is especially prominent in the easy condition of Task 2 . The response-monitoring hypothesis, however, assumes that Task-1 motor response lengthens the duration of Task 2 prior to response selection (i.e., the additional slack is inserted before Task- 2 response selection)

hypothesis was obtained with different contextual factors: (a) whether the researchers focused on the traditional PRP effect or the residual PRP effect, (b) whether the key dependent measures were behavioral (RT2, Bratzke et al., 2009) or include electrophysiological measures (LRP, Jentzsch et al., 2007), and (c) whether the primary cause of the responserelated interference (i.e., Task-1 motor response) was manipulated: Bratzke et al. (2009) manipulated the duration of Task-1 motor response while Jentzsch et al. (2007) did not. To help resolve the debate regarding the two hypotheses while addressing these methodological discrepancies, we directly manipulated the Task-1 motor requirement, focused on the traditional PRP effect (rather than the residual PRP effect), while obtaining both behavioral and electrophysiological measures at the same time.

\section{The present study}

The purpose of the present study is to test the two competing hypotheses regarding the nature of response-related interference. We manipulated the following two factors that should differentially affect Task 2 in terms of both RT2 and LRP according to the two hypotheses: (a) the primary cause of the response-related interference: whether Task 1 requires a motor response (Go vs. NoGo) and (b) Task-2 
response-selection difficulty (compatible vs. non-compatible stimulus-response mapping).

At long SOAs (900 ms in Experiment 1 and $1200 \mathrm{~ms}$ in Experiment 2), which have minimal response-related interference, both hypotheses assume that Task 2 is performed with little influence from the Task-1 motor requirement. Therefore, at long SOAs, there should be an observable Task-2 difficulty effect on RT2 and LRPs regardless of Task-1 motor requirement. That is, RT2 and S2-LRP (the premotoric duration) should be longer in the difficult condition than in the easy condition, while the LRP-R2 (the motoric duration) should be independent of Task-2 difficulty as well as Task-1 motor requirement.

At the short SOA, which has maximal response-related interference, the two hypotheses predict differential effects of Task-1 motor requirement on RT2 and LRPs. Regarding RT2, the most distinguishable prediction between the two hypotheses is, as discussed earlier, whether the Task-2 difficulty effect is reduced when Task 1 requires a motor response. According to the motor-bottleneck hypothesis, Task-1 motor processing creates the response-related bottleneck after the response-selection stage of Task 2 (b in Fig. 3). Therefore, it predicts absorption of the Task-2 difficulty effect into the slack when Task 1 requires a motor response (Go trials) but not when Task 1 does not require a response (NoGo trials), resulting in a two-way interaction between Task-1 motor requirement and Task-2 difficulty on RT2 (compare $a$ and $b$ in Fig. 3). In contrast, the responsemonitoring hypothesis asserts that Task-1 motor response generates the response-related bottleneck before the response-selection stage of Task 2 (c in Fig. 3). Therefore, it predicts a consistent lengthening of RT2 due to Task-1 motor response in both the easy and difficult conditions of Task 2-no interaction between Task-1 motor requirement and Task-2 difficulty (compare a and c in Fig. 3). Figure 4 shows the predictions of the two hypotheses on RT2.

In addition, the motor-bottleneck hypothesis predicts a three-way interaction between Task-1 motor requirement, Task-2 difficulty, and SOA: the two-way interaction between Task-1 motor requirement and Task-2 difficulty will be observed at the short SOA but not at the long SOA. In contrast, the response-monitoring hypothesis does not predict such a three-way interaction.

The two competing hypotheses also make different predictions regarding the premotoric duration of Task 2 (as measured by the S2-LRP) at the short SOA. Because the motor-bottleneck hypothesis assumes that the Task-1 motor response inserts the additional slack after the response selection of Task 2, the hypothesis predicts a consistent premotoric duration of Task 2 regardless of Task-1 motor requirement (i.e., compare $\mathrm{a}$ and $\mathrm{b}$ in Fig. 3). In contrast, because the response-monitoring hypothesis assumes that the response-related bottleneck occurs before the response-selection stage of Task 2, Task-1 motor response lengthens the premotoric durations of Task 2 regardless of Task-2 difficulty (compare a and c). Neither hypothesis predicts a significant three-way interaction on S2-LRP across the three factors because they both assume consistent Task-2 difficulty effect across conditions.

Finally, regarding the motoric duration of Task 2 (as measured by LRP-R2), both hypotheses predict that it should be invariant across conditions, with just one exception: the motor-bottleneck hypothesis predicts a lengthening of LRP-R2 when Task 2 is easy, the SOA is short, and Task 1 requires a motor response. This exception results from the motor-bottleneck hypothesis' assumption that, in this specific condition, the response-selection stage of Task 2 , during which the LRP begins, is first followed by the motor refractory period and then followed by the motor response (see the easy condition of Task 2 in b in Fig. 3). Table 1 summarizes the predictions of the two hypotheses with respect to RT2, S2-LRP, and LPR-R2.

\section{Experiment 1}

\section{Method}

\section{Participants}

Twenty-seven undergraduate students from Kennesaw State University and the first two authors of this paper participated in Experiment 1. For the LRP analysis, five participants were excluded due to excessive noise on either electrode $\mathrm{C} 3$ or $\mathrm{C} 4$ which precluded calculation of the LRP (their behavioral data were included in RT and ACC analysis). Another was lost due to excessive EEG noise on all channels on approximately half of all trials. Another two participants were excluded because of their low accuracy (lower than $80 \%$ for either Task 1 or Task 2). Among the remaining participants (ten women, eleven men, $M_{\text {age }}=23.57$, age range $=18-46$ ), seven participants were African American, eleven were White (non-Hispanic), two were Asian, and one reported multiple ethnicities. Three reported being left-handed and all reported normal or corrected-to-normal vision.

\section{Stimuli and apparatus}

The auditory stimuli of Task 1 (S1) were three sinusoidal tones of 800,1000 , and $1200 \mathrm{~Hz}$. The visual stimuli of Task 2 (S2) were arrows pointing either left, up, right, or down (approximately $2-\mathrm{cm}$ length and 0.6- $\mathrm{cm}$ width with the viewing distance of about $70 \mathrm{~cm}$ ). On each trial, one of the three tones was presented over two speakers located on either side of the monitor in front of the participant and one of the four arrows was presented in the middle of the 


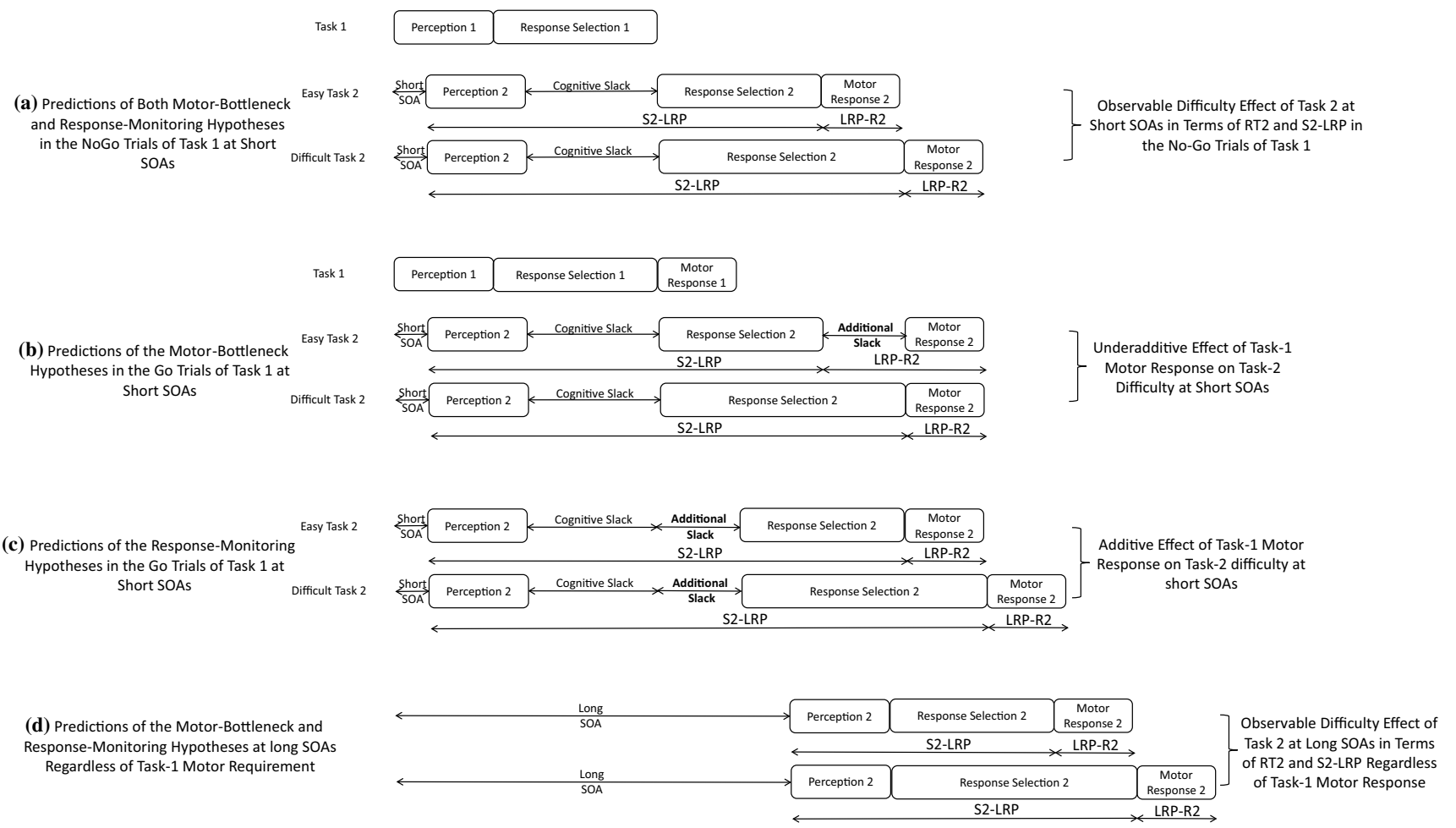

Fig. 3 Predictions of the motor-bottleneck and response-monitoring hypotheses as a function of Task-1 motor requirement, Task- 2 difficulty, and stimulus-onset asynchrony (SOA). According to the motor-bottleneck hypothesis, the response-related bottleneck (causing the additional slack) that occurs after the response selection of Task 2 can absorb the Task- 2 difficulty effect in the Go trials but not in the NoGo trials at short SOAs (compare $\mathbf{a}, \mathbf{b}$, and $\mathbf{d}$ ). In contrast, the response-monitoring hypothesis assumes that the additional slack is located before the response selection of Task 2 and cannot absorb

monitor. Participants pressed the $z, x,>$, and ? keys located at the corners of the second-bottom row of a keyboard to respond. They rested the left middle, left index, right index, right middle fingers on those four keys, respectively. The second author programmed the experiment in $\mathrm{C}$ using functions of the Allegro open source game library and compiled with MinGW. Stimuli were controlled by a desktop Windows PC. ${ }^{1}$

We recorded the electroencephalogram (EEG) using a 40-channel NuAmps amplifier (Neuroscan Compumedics, Inc.) sampling at $1000 \mathrm{~Hz}$ with a Quik-Cap for electrode placement while keeping electrode impedances below $10 \mathrm{k} \Omega$. We processed the signals using EEGLAB 4.51 (Delorme \& Makeig, 2004). We downsampled data to $500 \mathrm{~Hz}$ and filtered with a $0.1-\mathrm{Hz}$ high-pass and $45-\mathrm{Hz}$ low-pass filter. Data were epoched relative to both stimuli

\footnotetext{
${ }^{1}$ Source code and the datasets generated and analyzed during the current study are available in the following repository http://facul tyweb.kennesaw.edu/kjung2/research.php.
}

Task-2 difficulty effect regardless of Task- 1 motor requirement (panel c). Regarding the premotoric and motoric durations of Task 2, the motor-bottleneck hypothesis predicts a consistent premotoric duration (S2-LRP) regardless of Task-1 motor requirement, along with a lengthened motoric duration (LRP-R2) due to Task-1 motor response only in the easy condition of Task 2 at short SOAs (a, b). Meanwhile, the response-monitoring hypothesis predicts a lengthened premotoric duration of Task 2 in the Go trials compared to the NoGo trials at short SOAs along with a consistent motoric duration $(\mathbf{a}, \mathbf{c})$

and responses using only trials with correct responses. We removed data for bad channels or epochs of excessive noise based on visual inspection. For eye blink and other artifacts, we used an independent component analysis approach (Delorme \& Makeig, 2004). After removing the artifacts, we reconstructed the EEG. To calculate the LRP, event-related potential (ERP) data were further filtered with a $4-\mathrm{Hz}$ lowpass. Stimulus-locked averages were taken from $500 \mathrm{~ms}$ pre-stimulus to $1500 \mathrm{~ms}$ post-stimulus with the pre-stimulus epoch serving as a baseline. Response-locked averages were taken from $1000 \mathrm{~ms}$ before to $1000 \mathrm{~ms}$ after the response, with a 100-ms baseline from 1000 to $900 \mathrm{~ms}$ before the response. Averages were calculated separately for each condition and side of response. The LRP was then calculated according to Coles's (1989) formula: LRP $=[C 3-C 4$ (right response) $+\mathrm{C} 4-\mathrm{C} 3$ (left response)]/2. The S-LRP peak was then defined as the minimum in the epoch $500 \mathrm{~ms}$ prior to the mean response time, while the LRP-R peak was defined as the minimum in the $500 \mathrm{~ms}$ prior to the response. To estimate LRP onset, we used the jackknife procedure 
Fig. 4 Predictions of the motor-bottleneck and responsemonitoring hypotheses on Task-2 response time (RT2) as a function of Task-1 motor requirement, Task-2 difficulty, and stimulus-onset asynchrony (SOA). a According to the motor-bottleneck hypothesis, at short SOAs, Task-2 difficulty effect should be reduced more strongly in the Go trials of Task 1 than in the NoGo trials. b According to the responsemonitoring hypothesis, at short SOAs, RT2 should be increased in the Go trials of Task 1 relative to the NoGo trials, and this increase should be similar for the easy and difficult conditions

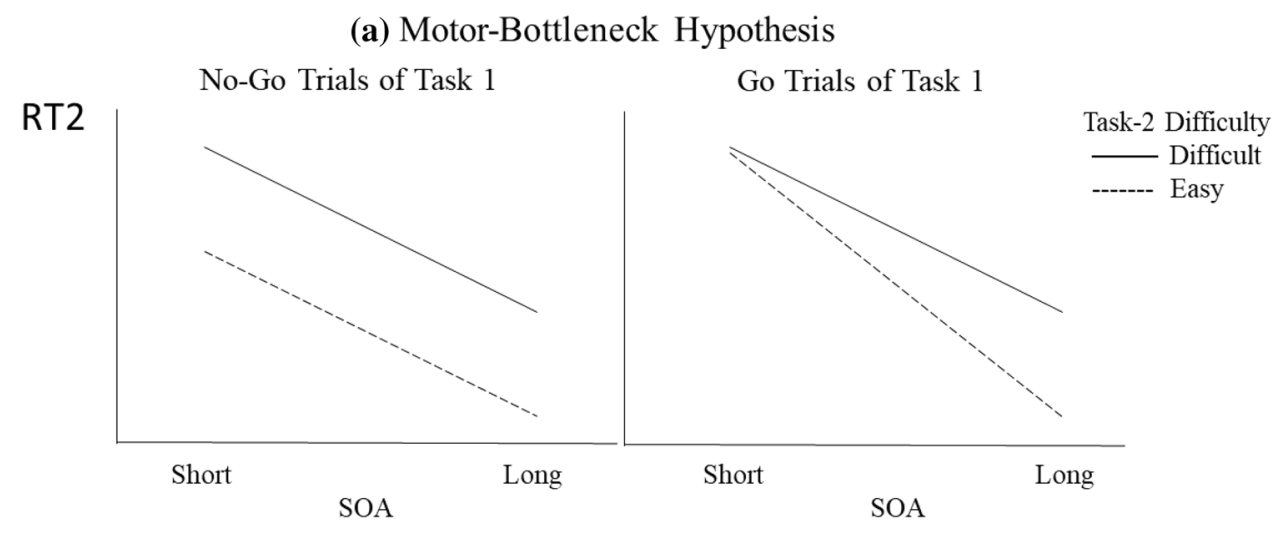

(b) Response-Monitoring Hypothesis

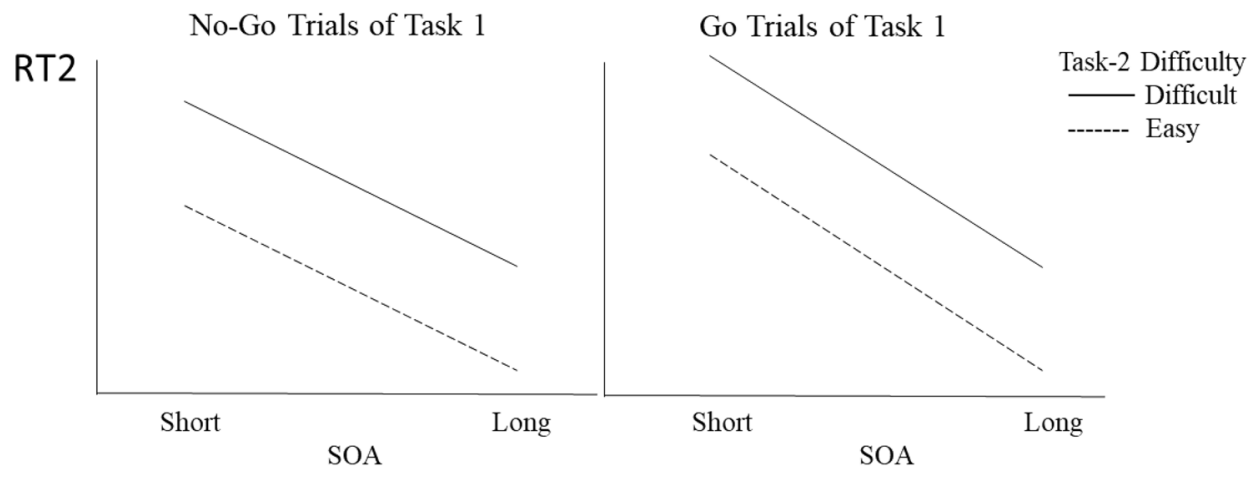

Table 1 Summary of predictions of the motor-bottleneck hypothesis and the response-monitoring hypothesis for the short stimulus-onset asynchrony (SOA) with respect to Task-2 mean response time (RT2), S2-LRP, and LRP-R2

\begin{tabular}{|c|c|c|c|}
\hline \multirow[t]{2}{*}{ Hypotheses and results } & \multicolumn{3}{|l|}{ Predictions } \\
\hline & RT2 & S2-LRP & LRP-R2 \\
\hline The motor-bottleneck hypothesis & $\begin{array}{l}\text { Reduced Task-2 difficulty effect } \\
\text { when Task-1 requires a motor } \\
\text { response (Go trials) relative to } \\
\text { when it does not (NoGo trials) }\end{array}$ & $\begin{array}{l}\text { Consistent effect of Task-2 } \\
\text { difficulty regardless of } \\
\text { Task-1 motor requirement }\end{array}$ & $\begin{array}{l}\text { LRP-R2 in the easy condition of } \\
\text { Task } 2 \text { is longer when Task- } 1 \\
\text { requires a response (Go trials) } \\
\text { than when it does not (NoGo } \\
\text { Trials) }\end{array}$ \\
\hline The response-monitoring hypothesis & $\begin{array}{l}\text { Consistent Task-2 difficulty effect } \\
\text { regardless of Task-1 motor require- } \\
\text { ment }\end{array}$ & $\begin{array}{l}\text { Main effect of Task-1 motor } \\
\text { requirement (longer } \\
\text { S2-LRP by Task-1 motor } \\
\text { response) }\end{array}$ & Consistent LRP-R2 \\
\hline
\end{tabular}

of Miller, Patterson, and Ulrich (1998) instead of the segmented regression approaches (Mordkoff \& Gianaros, 2000; Schwarzenau, Falkenstein, Hoormann, \& Hohnsbein, 1998) because the jackknife procedure is particularly robust to low signal-noise ratio (Ulrich \& Miller, 2001). In this procedure, several LRP waveforms were calculated, each time leaving out a single subject's waveform. To estimate LRP onset, the peak of the LRP was first identified. For the S-LRP, this was defined as the minimum amplitude (i.e., greatest negative deviation) in the $500 \mathrm{~ms}$ preceding the average RT for that condition. For the LRP-R, the peak was defined as the minimum in the epoch $500 \mathrm{~ms}$ prior to the response. Then, the LRP onset was determined by moving back in time from the peak until the point at which the waveform first reached $50 \%$ (S-LRP) or $90 \%$ (LRP-R) of the peak amplitude, following the suggestion of Miller et al. After removal of error, grouping, and outlier trials, the least number of trials for any subject in any condition was 27 (in the difficult condition of Task 2, NoGo Task 1, and at the short SOA). 


\section{Design and procedure}

A fixation dot $(0.25 \mathrm{~cm} \times 0.25 \mathrm{~cm})$ was presented in the middle of the screen and was present at all times except when error feedback was given or it was covered by S2 (arrow target). At the beginning of each trial, S1 (a tone) was presented for $100 \mathrm{~ms}$. After one of two SOAs (100 or $900 \mathrm{~ms}$ ), S2 (an arrow) was presented for $500 \mathrm{~ms}$, after which a response period lasted up to $3000 \mathrm{~ms}$ or until a response to $\mathrm{S} 2$ was emitted. Upon the response, a random inter-trial interval between 1 and $2 \mathrm{~s}$ was initiated.

In Task 1, participants were instructed to indicate whether the tone was low $(800 \mathrm{~Hz})$ or high $(1200 \mathrm{~Hz})$ by pressing the $x$ and $>$ keys using the index finger of each hand (Go trials). When $\mathrm{S} 1$ was a medium tone $(1000 \mathrm{~Hz})$, they were to withhold their response and perform only Task 2 (NoGo trials). In Task 2, participants were instructed to indicate the arrow's direction by pressing the $z$ and ? keys using the middle finger of each hand. For the horizontal arrows, the stimulus-response mapping was compatible (the easy condition of Task 2): the left arrow corresponded to the left middle finger ( $z$ key) and the right arrow corresponded to the right middle finger (? key). For the vertical arrows, the mapping was noncompatible (the difficult condition of Task 2): the vertical arrows required presses of horizontally aligned keys. The non-compatible mapping was counterbalanced across participants so that some participants were given the up = right/ down $=$ left mapping and others the up $=$ left $/$ down $=$ right mapping.

Participants were instructed to respond to both tasks as quickly and accurately as possible with priority placed on Task 1. Feedback was presented immediately after the response to S2. If the Task-1 response was incorrect, the message, for example, "ERROR. REMEMBER X for LOW, > for HIGH! No response for the MIDDLE tone!" was presented for $3 \mathrm{~s}$, with the message tailored to the tone-key mapping. If the Task-2 response was incorrect, the message "ERROR. REMEMBER Z FOR LEFT OR UP ARROW, I KEY FOR RIGHT OR DOWN ARROW" was presented for $3 \mathrm{~s}$, with the message tailored to the mapping between the arrows and keys. If both responses were incorrect on a trial, the Task- 1 feedback was given for $3 \mathrm{~s}$, followed immediately by the Task- 2 feedback for an additional $3 \mathrm{~s}$. No feedback was given for correct trials.

There was a total of 672 trials (three tones $\times$ two SOAs $\times$ four arrows $\times 28$ repetition; the order of these trials was completely randomized). Before the main experimental block, participants performed a tone-only practice block of 24 trials, an arrow-only practice block of 24 trials, and dual-task practice block of 48 trials. The experiment lasted approximately $2 \mathrm{~h}$, including preparation, and participants took a break in the middle of the experiment.

\section{Results}

We excluded the practice trials and the first 10 of the experimental trials from data analysis. We excluded trials if RT1 or RT2 was below or above 2.5 standard deviations from that participant's mean ( $3 \%$ of all trials). We also removed trials ( $2 \%$ of all trials) consistent with response grouping, defined as inter-response-interval $\leq 100 \mathrm{~ms}$ (see Ulrich \& Miller, 2008). Finally, we removed trials from RT and ERP analysis if the responses were incorrect for either Task 1 or Task 2 (15\% of all trials). The resulting mean RTs and accuracies (ACCs) for Task 1 and Task 2 are shown in Table 2.

\section{RT analysis}

A repeated-measures analysis of variance (ANOVA) was conducted with the factors of Task- 1 motor requirement (Go vs. NoGo), Task-2 difficulty (easy vs. difficult), and SOA (100 ms vs. $900 \mathrm{~ms}$ ) on RT1 (Go trials only) and RT2. Regarding RT1, there was a main effect of SOA, $F(1$, $25)=5.863, p=0.023, \eta_{p}^{2}=0.190$ : RT1 was $68 \mathrm{~ms}$ longer at the long SOA than at the short SOA. There was also a main effect of Task-2 difficulty on RT1, $F(1,25)=6.763$, $p=0.015, \eta_{p}^{2}=0.213$ : RT1 was $40 \mathrm{~ms}$ longer in the difficult condition of Task 2 than in the easy condition. However, SOA and Task-2 difficulty did not show a significant interaction effect on RT1, $F(1,25)=2.097, p=0.160, \eta_{p}^{2}=0.077$.

RT2 was greater at the short SOA $(1380 \mathrm{~ms})$ than at the long SOA ( $875 \mathrm{~ms})$ by $505 \mathrm{~ms}$, showing the typical PRP effect (see the upper panels in Fig. 5), $F(1,25)=527.815$, $p<0.001, \eta_{p}^{2}=0.955$. According to the response-selection bottleneck hypothesis (Pashler, 1984, 1994; Welford, 1952), such an increase of RT2 at the short SOA is due entirely to the postponement of the response selection of Task 2 during Task-1 response selection (i.e., the cognitive slack), without any motor-related interference. Contrary to this assumption, the need for a Task-1 motor response lengthened RT2 by $86 \mathrm{~ms}$ : RT2 was slower in the Go trials $(1170 \mathrm{~ms})$ than in the NoGo trials $(1085 \mathrm{~ms}), F(1,25)=19.224, p<0.001$, $\eta_{p}^{2}=0.435$, supporting the hypothesis of response-related interference. As predicted by both hypotheses being tested, such an effect of Task-1 motor requirement on RT2 was more prominent at the short SOA $(110 \mathrm{~ms})$ than at the long $\mathrm{SOA}(62 \mathrm{~ms}), F(1,25)=5.320, p=0.030, \eta_{p}^{2}=0.175$. Looking at this differently, the overall delay of RT2 at the short SOA relative to the long SOA (the PRP effect) was $481 \mathrm{~ms}$ in the NoGo trials while it was $529 \mathrm{~ms}$ in the Go trials. That is, when Task 1 requires a motor response, the PRP effect is even greater, suggesting that response-related interference contributes to the PRP effect that typically involves Task-1 motor response. 
Table 2 Mean response time (RT, in milliseconds) and accuracy (ACC, in percentages) for Task 1 and 2 of Experiment 1 and 2 as a function of Task-1 motor-response requirement (Go vs. NoGo), Task-2 difficulty (easy vs. difficult), and stimulus-onset asynchrony (SOA; short vs. long)

\begin{tabular}{|c|c|c|c|c|c|c|c|}
\hline & \multirow[t]{3}{*}{ Task } & \multirow{3}{*}{$\begin{array}{l}\text { Task-1 } \\
\text { Motor response }\end{array}$} & \multirow{3}{*}{$\begin{array}{l}\text { Task-2 } \\
\text { Difficulty }\end{array}$} & \multicolumn{4}{|l|}{ SOA } \\
\hline & & & & \multicolumn{2}{|c|}{ Short (100 ms) } & \multicolumn{2}{|c|}{$\begin{array}{l}\text { Long }(900 \mathrm{~ms} \text { in } \\
\text { Experiment } 1 \text {; } \\
1200 \mathrm{~ms} \text { in Experi- } \\
\text { ment } 2 \text { ) }\end{array}$} \\
\hline & & & & RT (ms) & $\mathrm{ACC}(\%)$ & RT (ms) & $\operatorname{ACC}(\%)$ \\
\hline \multirow[t]{8}{*}{ Experiment 1} & \multirow[t]{4}{*}{ Task 1} & \multirow[t]{2}{*}{ Go } & Easy & 1118 & 85 & 1199 & 91 \\
\hline & & & Difficult & 1171 & 91 & 1226 & 93 \\
\hline & & \multirow[t]{2}{*}{ NoGo } & Easy & - & 91 & - & 93 \\
\hline & & & Difficult & - & 90 & - & 92 \\
\hline & \multirow[t]{4}{*}{ Task 2} & \multirow[t]{2}{*}{ Go } & Easy & 1342 & 92 & 790 & 98 \\
\hline & & & Difficult & 1527 & 89 & 1021 & 91 \\
\hline & & \multirow[t]{2}{*}{ NoGo } & Easy & 1164 & 92 & 692 & 99 \\
\hline & & & Difficult & 1486 & 88 & 996 & 92 \\
\hline \multirow[t]{8}{*}{ Experiment 2} & \multirow[t]{4}{*}{ Task 1} & \multirow[t]{2}{*}{ Go } & Easy & 1007 & 90 & 1003 & 96 \\
\hline & & & Difficult & 1044 & 96 & 980 & 96 \\
\hline & & \multirow[t]{2}{*}{ NoGo } & Easy & - & 94 & - & 97 \\
\hline & & & Difficult & - & 95 & - & 97 \\
\hline & \multirow[t]{4}{*}{ Task 2} & \multirow[t]{2}{*}{ Go } & Easy & 1301 & 93 & 583 & 98 \\
\hline & & & Difficult & 1468 & 92 & 816 & 92 \\
\hline & & \multirow[t]{2}{*}{ NoGo } & Easy & 1173 & 92 & 561 & 100 \\
\hline & & & Difficult & 1459 & 91 & 852 & 93 \\
\hline
\end{tabular}

The data confirmed that our manipulation of Task-2 difficulty was successful: at the long SOA, RT2 was $268 \mathrm{~ms}$ greater in the non-compatible stimulus-response condition (difficult condition: $1009 \mathrm{~ms}$ ) than in the compatible condition (easy condition: $741 \mathrm{~ms}$ ), $F(1,25)=151.162, p<0.001$, $\eta_{p}^{2}=0.858$. As discussed earlier (see also Table 1 ), the critical test between the motor-bottleneck and the responsemonitoring hypotheses concerns whether such a Task-2 difficulty effect is reduced at the short SOA when Task 1 requires a motor response and whether there is a three-way interaction across the three experimental factors (the twoway interaction between Task-1 motor requirement and Task-2 difficulty appears at the short SOA but not at the long SOA). Looking at the short SOA only, the comparison between Go trials and NoGo trials with respect to the Task-2 difficulty effect showed that the difficulty effect was significantly smaller in the Go trials $(185 \mathrm{~ms})$ than in the NoGo trials $(332 \mathrm{~ms}), F(1,25)=39.721, p<0.001, \eta_{p}^{2}=0.614$. Such a reduction in the Task-2 difficulty effect by the Task-1 motor response indicates that the response-related bottleneck occurs after the response-selection stage of Task 2, consistent with the motor-bottleneck hypothesis; in contrast, the response-monitoring hypothesis does not allow such a reduction because of the assumption that Task-1 motor requirement affects the stages before the response selection of Task 2. Looking at the long SOA, there was another significant interaction between Task-1 motor requirement and Task-2 difficulty, $F(1,25)=12.227, p=0.002, \eta_{p}^{2}=0.328$ : similar to the interaction pattern at the short SOA, the difficulty effect was significantly smaller in the Go trials $(231 \mathrm{~ms})$ than in the NoGo trials (304 ms). Although the significant threeway interaction across Task-1 motor requirement, Task- 2 difficulty, and SOA indicated that this latter interaction at the long SOA (reduced Task-2 difficulty due to Task-1 motor response) was not as strong as the one at the short SOA, $F(1,25)=4.846, p=0.037, \eta_{p}^{2}=0.162$, such a modulation of Task-2 difficulty by Task-1 motor requirement even at the long SOA was somewhat unexpected. Both hypotheses predicted a significant difficulty effect at the long SOA regardless of the Task-1 motor requirement. We discussed the possible cause the Task- 2 difficulty modulation by Task-1 motor requirement even at the long SOA in the discussion section of this experiment.

\section{Accuracy (ACC) analysis}

Task-1 accuracy (ACC1) was significantly lower at the short SOA than at the long SOA $(89 \%$ vs. $92 \%), F(1,25)=43.938$, $p<0.001, \eta_{p}^{2}=0.637$. ACC1 was greater when Task 2 was difficult $(92 \%)$ than when it was easy $(90 \%), F(1$, $25)=11.840, p=0.002, \eta_{p}^{2}=0.321$. The effect of Task-2 difficulty on ACC1 was greater at the short SOA $(-3 \%)$ than at the long SOA $(-1 \%), F(1,25)=4.509, p=0.044$, $\eta_{p}^{2}=0.153$. The difficulty effect was also greater in the Go trials $(-4 \%)$ than in the NoGo trials $(1 \%), F(1,25)=18.821$, $p<0.001, \eta_{p}^{2}=0.429$. There was also a significant three-way 
Fig. 5 Response time to Task 2 (RT2) as a function of Task-1 motor requirement, Task-2 difficulty, and stimulus-onset asynchrony (SOA) in Experiment 1 and Experiment 2. Error bars show the $95 \%$ confidence interval
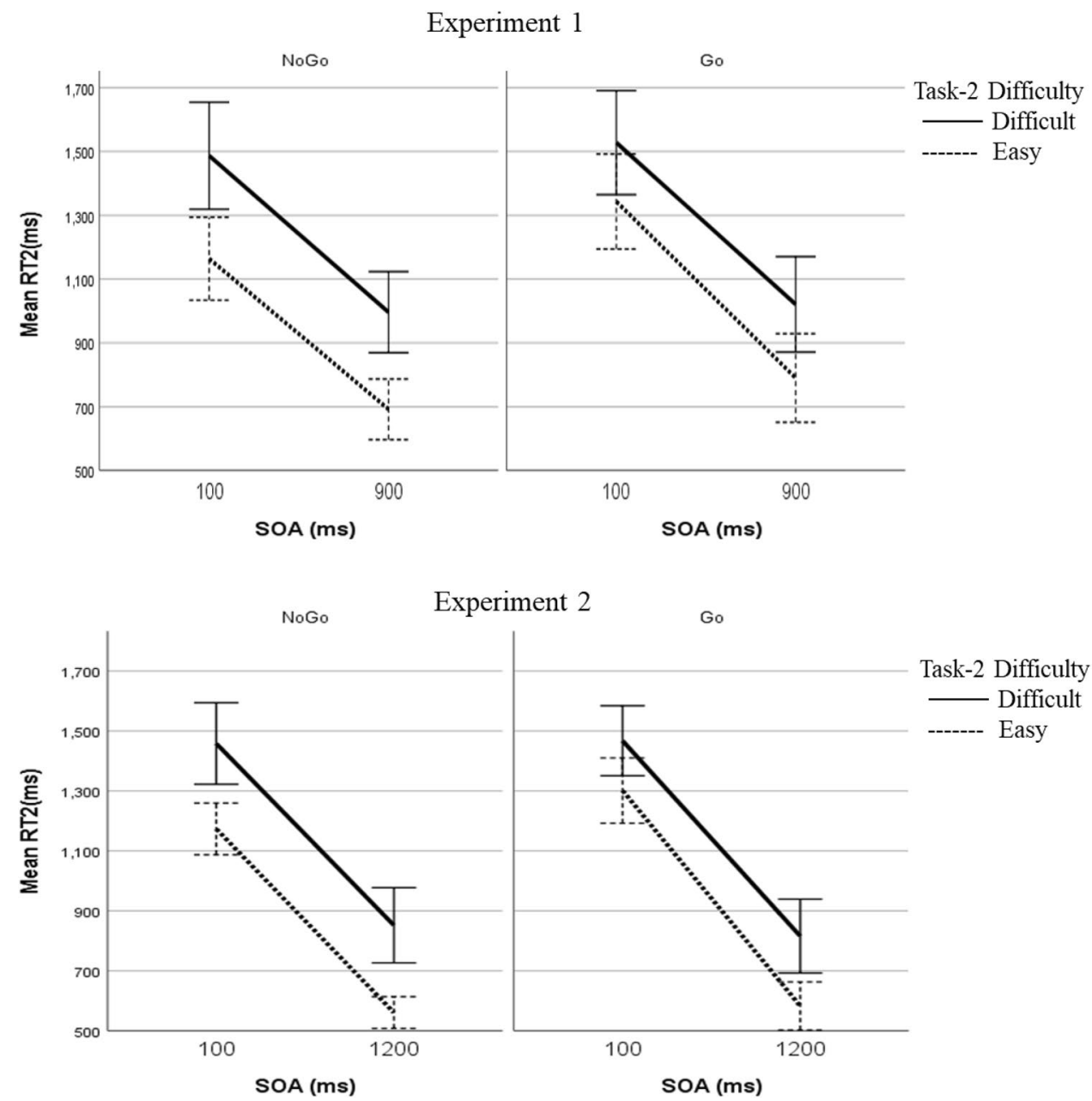

interaction across the three experimental factors, indicating that the Task-2 difficulty effect on ACC1 was greater at the short SOA than at the long SOA but only in the Go trials $(-4 \%)$, while it was the same across SOAs in the NoGo trials $(1 \%), F(1,25)=6.532, p=0.017, \eta_{p}^{2}=0.207$.

Task-2 accuracy (ACC2) was significantly lower at the short SOA than at the long SOA (90\% vs. 95\%), $F(1$, $25)=54.060, p<0.001, \eta_{p}^{2}=0.684$. Task-2 difficulty manipulation showed the expected effect on ACC2: $95 \%$ vs. $90 \%$ in the compatible and non-compatible conditions, $F(1,25)=28.842, p<0.001, \eta_{p}^{2}=0.536$. There was a significant interaction between Task-2 difficulty and SOA, $F(1$, $25)=8.495, p=0.007, \eta_{p}^{2}=0.254$, suggesting that the difficulty effect was smaller at the short SOA (4\%) than at the long SOA (7\%). However, Task-1 motor requirement did not show any significant main or interaction effects on ACC2 $(p s>0.05)$.

\section{LRP analysis}

Grand averaged LRPs for Task 2 are illustrated in Fig. 6. The S2-LRP is shown in Fig. 7 as a function of SOA, Task-1 motor requirement, and Task-2 difficulty. The mean onsets of S2-LRP and LRP-R2 of Task 2 are reported in Table 3.

Regarding the S2-LRP, the main effect of SOA was significant $(p=0.012)$ : the onset of the S2-LRP was delayed at the short SOA (800 ms) relative to the long SOA (424 ms), indicating a lengthening of the premotoric duration of Task 2 as task overlap increases, consistent with the cognitive slack of the response-selection bottleneck hypothesis (Osman \& Moore, 1993; Pashler, 1984, 1994). The main effect of task difficulty on S2-LRP was also significant $(p=0.022)$. The onset was delayed in the difficult condition $(777 \mathrm{~ms})$ relative to the easy condition $(448 \mathrm{~ms})$. No other effects or interactions were significant $(p>0.05)$. Notably, unlike what the response-monitoring hypothesis predicted, there was no significant main effect of Task-1 motor requirement on S2-LRP onset at the short SOA; furthermore, while the response-monitoring hypothesis predicted a consistent delay of S2-LRP due to Task-1 motor response at the short SOA, the S2-LRP onset in the difficult condition occurred (at least numerically) earlier in the Go trials $(860 \mathrm{~ms})$ than in the NoGo trials (1072 ms). 

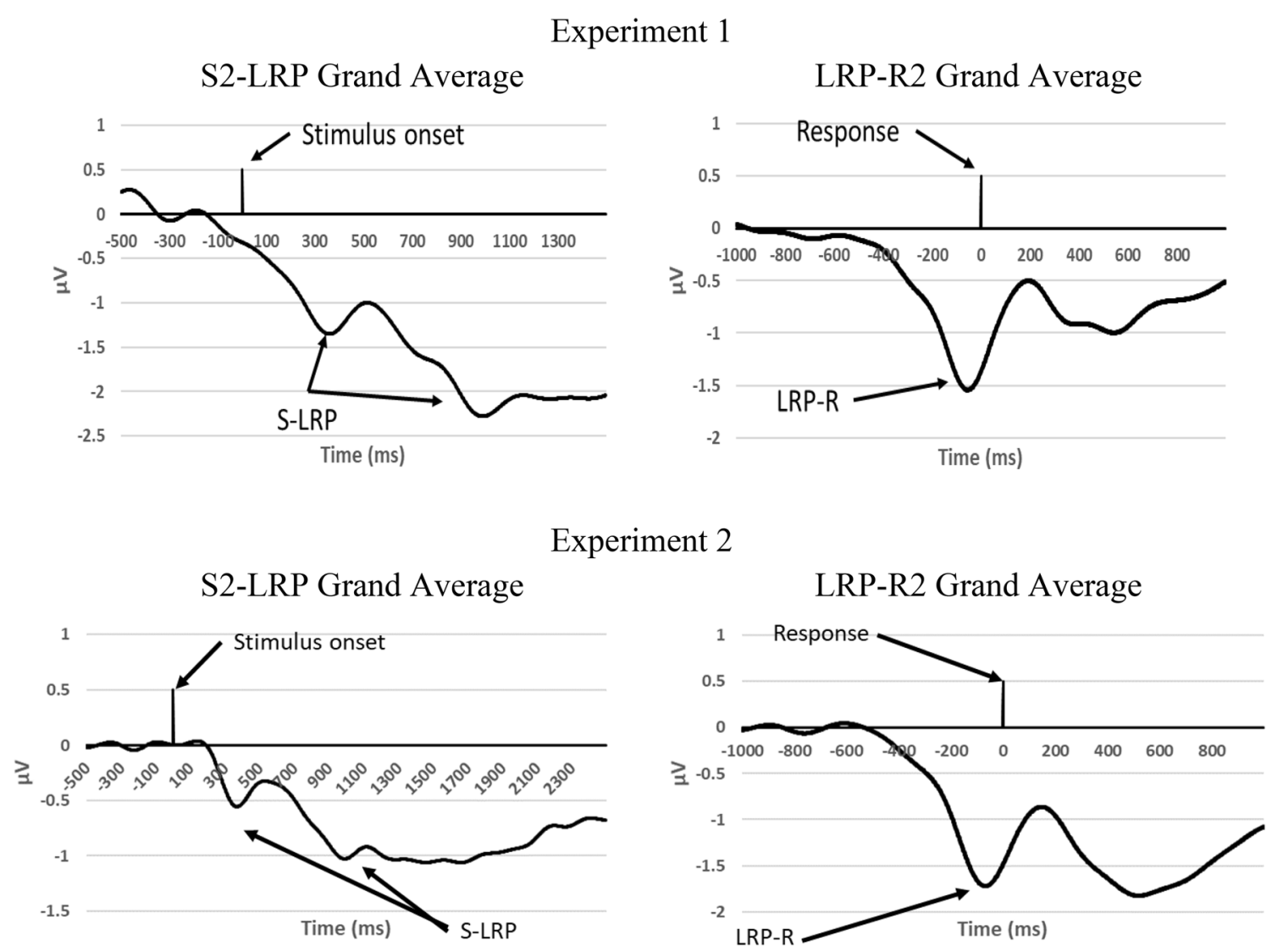

Fig. 6 Grand averaged S2-LRP and LRP-R2 of Task 2 in Experiment 1 and Experiment 2. Negative voltages are plotted downward

The results did not match one of the predictions of the motor-bottleneck hypothesis. This hypothesis predicted a selective lengthening of LRP-R2 when Task 1 required a motor response for the easy condition of Task 2 , at the short SOA. However, none of the effects on LRP-R2 onset were significant ( $p s>0.05$ ). If anything, LRP-R2 occurred later $(-96 \mathrm{~ms})$ in this specific condition than in other conditions (e.g., $-114 \mathrm{~ms}$ in the difficult condition of Task 2, at the short SOA, when Task 1 required a motor response; see Table 3).

\section{Discussion}

Overall, the results better matched the predictions of the motor-bottleneck hypothesis than those of the responsemonitoring hypothesis, which are summarized in Table 1. First, the Task-2 difficulty effect on RT2 was reduced with a Task-1 motor response. This suggests that the responserelated bottleneck delays the motor stage of Task 2 , as asserted by the motor-bottleneck hypothesis, rather than delaying the response-selection stage of Task 2 as asserted by the response-monitoring hypothesis. In addition, there was a three-way interaction across the three experimental factors, which is more consistent with the motor-bottleneck hypothesis, although the nature of the interaction was not exactly what the motor-bottleneck hypothesis predicted, as discussed below. Furthermore, the relatively consistent premotoric duration of Task 2 (as measured by S2-LRP) regardless of Task-1 motor requirement supports the motor-bottleneck hypothesis, which places the locus of the response-related bottleneck after the response-selection stage of Task 2; in contrast, the response-monitoring hypothesis predicts a systematic lengthening of the premotoric duration when Task 1 requires a motor response. However, we should note that the last prediction of the motor-bottleneck hypothesis-selective lengthening of LRP-R2 in the easy condition of Task 2 with Task-1 motor response at the short SOA-was not supported.

Although the overall results were more consistent with the motor-bottleneck hypothesis, there were some effects that were not predicted by either hypothesis, especially at the long SOA. For example, as reported earlier, at the long SOA, the comparison between Go trials and NoGo trials showed that the difficulty effect was significantly smaller in the Go trials $(231 \mathrm{~ms})$ than in the NoGo trials (304 ms). Neither hypothesis predicts such a reduced Task- 2 difficulty effect 


\section{Experiment 1}

(a)

a)
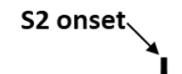

SOA

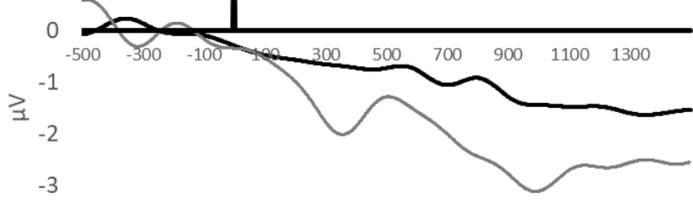

Time (ms)

$-100 \mathrm{~ms} \mathrm{SOA}-900 \mathrm{~ms} \mathrm{SOA}$

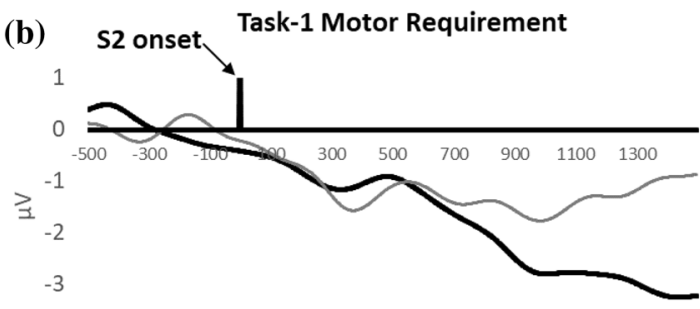

Time (ms)

Go - No-Go

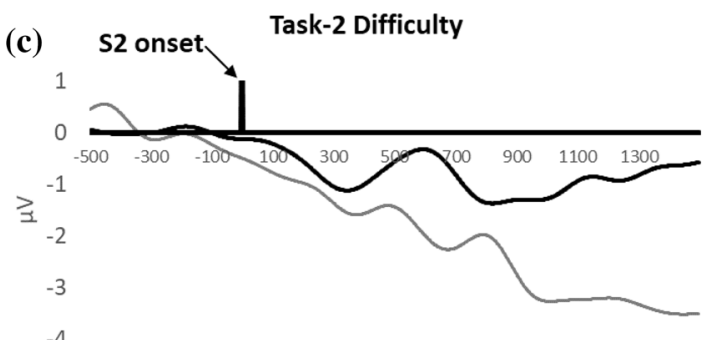

Time (ms)

—Easy —Difficult
Experiment 2

(d) S2 onset SOA

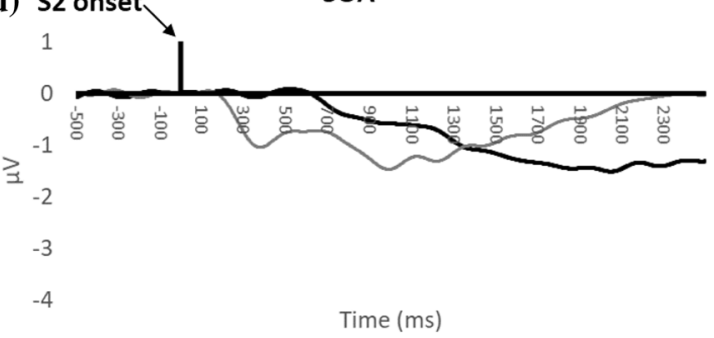

$-100 \mathrm{msSOA}-900 \mathrm{~ms} \mathrm{SOA}$

(e) S2 onset Task-1 Motor Requirement
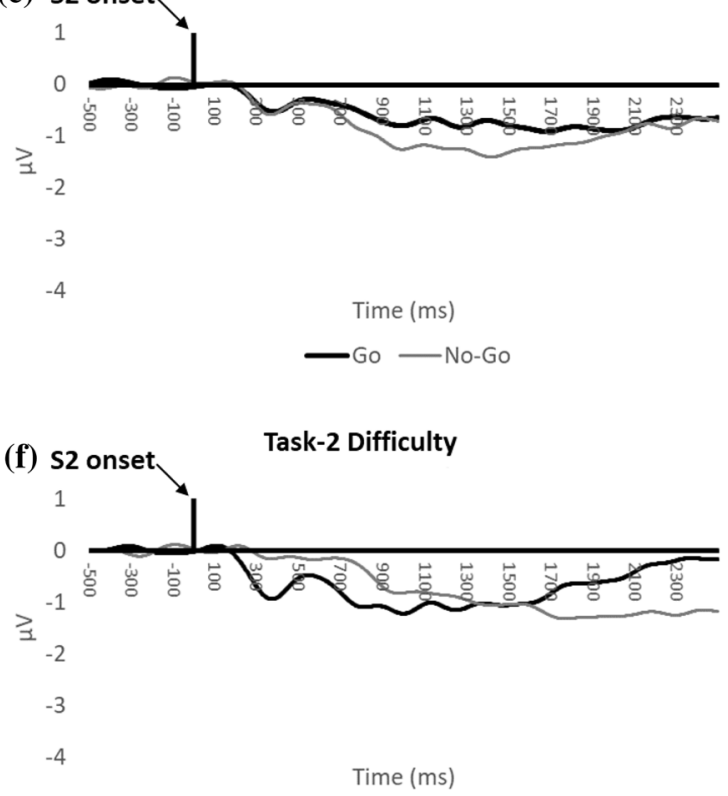

—Easy —Difficult

Fig. 7 Task-2 S2-LRP in Experiment 1 and Experiment 2. Grand averages are shown as a function of stimulus-onset asynchrony (SOA; a, d), Task-1 motor requirement $(\mathbf{b}, \mathbf{e})$, and Task-2 difficulty $(\mathbf{c}, \mathbf{f})$

Table 3 Mean onset of S2-LRP and LRP-R2 for Task 2 (in milliseconds) as a function of Task-1 motor-response requirement (Go vs. NoGo), Task-2 difficulty (easy vs. difficult), and stimulus-onset asynchrony (SOA; short vs. long) in Experiment 1 and 2

\begin{tabular}{|c|c|c|c|c|c|c|}
\hline & \multirow{3}{*}{$\begin{array}{l}\text { Task-1 } \\
\text { Motor response }\end{array}$} & \multirow{3}{*}{$\begin{array}{l}\text { Task-2 } \\
\text { Difficulty }\end{array}$} & \multicolumn{4}{|l|}{ SOA } \\
\hline & & & \multicolumn{2}{|c|}{ Short (100 ms) } & \multicolumn{2}{|c|}{$\begin{array}{l}\text { Long }(900 \mathrm{~ms} \text { in } \\
\text { Experiment } 1 ; 1200 \mathrm{~ms} \\
\text { in Experiment 2) }\end{array}$} \\
\hline & & & S2-LRP & LRP-R2 & S2-LRP & LRP-R2 \\
\hline \multirow[t]{4}{*}{ Experiment 1} & \multirow[t]{2}{*}{ Go } & Easy & 705 & -96 & 266 & -104 \\
\hline & & Difficult & 860 & -114 & 810 & -82 \\
\hline & \multirow[t]{2}{*}{ NoGo } & Easy & 564 & -110 & 255 & -90 \\
\hline & & Difficult & 1072 & -117 & 364 & -122 \\
\hline \multirow[t]{4}{*}{ Experiment 2} & \multirow[t]{2}{*}{ Go } & Easy & 910 & -111 & 230 & -119 \\
\hline & & Difficult & 1365 & -106 & 302 & -95 \\
\hline & \multirow[t]{2}{*}{ NoGo } & Easy & 632 & -165 & 228 & -134 \\
\hline & & Difficult & 1168 & -122 & 706 & -109 \\
\hline
\end{tabular}


due to Task-1 motor response at the long SOA. As another example of unexpected results at the long SOA, there was a significant delay of RT2 (by $62 \mathrm{~ms}$ ) in the Go trials relative to NoGo trials-response-related interference even at the long SOA $-F(1,25)=5.641, p=0.026, \eta_{p}^{2}=0.184$. However, again, neither hypothesis assumed that Task 2 would be modulated by Task-1 motor requirement at the long SOA.

We speculate that these unexpected results at the long SOA were due to residual processing overlap between the two tasks even in the long-SOA condition employed in Experiment 1 . Specifically, although the SOA of $900 \mathrm{~ms}$ corresponds to the typical long-SOA condition in PRP studies, it was not sufficiently long in Experiment 1 considering that the mean RT1 in the Go trials was $1179 \mathrm{~ms}$. Therefore, even in the long-SOA condition, Task 1 was often not completed, yielding the unexpected results (i.e., reduced Task-2 difficulty effect due to Task- 1 motor response as well as the response-related interference). This speculation motivated us to replicate Experiment 1 with an even longer long-SOA condition in Experiment 2.

\section{Experiment 2}

Experiment 2 replicated Experiment 1 with a long SOA of $1200 \mathrm{~ms}$ rather than $900 \mathrm{~ms}$. With this longer SOA, we expected that Task 1 would show even less influence on Task 2 at the long SOA than it did in Experiment 1. Therefore, the overall effect of SOA on Task 2 should be greater than the one observed in Experiment 1 (i.e., a greater PRP effect). Similarly, we also expected that Task-1 motor requirement would show diminished influence on Task 2 relative to Experiment 1 primarily due to the lack of its effect at the new long-SOA condition. However, there should still be an observable effect of Task-1 motor requirement on Task 2 at the short SOA.

\section{Method}

\section{Participants}

Fifteen undergraduate students from Kennesaw State University participated for partial course credit. One participant was excluded because of low Task-1 accuracy (lower than $80 \%$ ). Among the remaining participants (12 women, two men, $M_{\text {age }}=21$, age range $=18-33$ ), two participants were African American, 11 were White (non-Hispanic), and one was Asian. Two reported being left-handed and all reported normal or corrected-to-normal vision.

\section{Stimuli and procedure}

Experiment 2 was identical to Experiment 1 except that the long SOA changed from 900 to $1200 \mathrm{~ms}$.

\section{Results}

\section{RT analysis}

Data were handled in the same manner as in Experiment 1. Regarding RT1, SOA and Task-2 difficulty did not have a main effect $(p s>0.05)$, although their interaction was significant, $F(1,13)=11.808, p=0.004, \eta_{p}^{2}=0.476$. Specifically, the effect of Task-2 difficulty on RT1 was reversed at the long SOA ( $-23 \mathrm{~ms}$ ) compared to the short SOA (37 ms).

There was a significant PRP effect-RT2 was greater at the short SOA $(1350 \mathrm{~ms})$ than at the long SOA $(703 \mathrm{~ms})$ by $647 \mathrm{~ms}, F(1,13)=1010, p<0.001, \eta_{p}^{2}=0.987$ (see Table 2 and lower panels in Fig. 5). As we had expected, the current PRP effect was greater than the one in Experiment 1 $(505 \mathrm{~ms}), F(1,38)=17.983, p<0.001, \eta_{p}^{2}=0.321$, suggesting that the new long-SOA condition further separated the two tasks than the corresponding condition did in Experiment 1 , minimizing the interferences between tasks at the long SOA.

Regarding the effect of Task-1 motor requirement, RT2 in the Go trials (1042 ms) was longer than RT2 in the NoGo trials $(1011 \mathrm{~ms})$ by $31 \mathrm{~ms}$, which was marginally significant, $F(1,13)=4.160, p=0.062, \eta_{p}^{2}=0.242$. As expected, in Experiment 2, the effect of Task-1 motor requirement on RT2 was smaller than the corresponding effect in Experiment 1 (86 ms) - it was less than half of the effect in Experiment 1 , which was a marginally significant reduction, $F(1$, $38)=3.593, p=0.066, \eta_{p}^{2}=0.086$. However, there was still a significant effect of Task-1 motor requirement on RT2 at the short SOA $(69 \mathrm{~ms}), F(1,13)=13.975, p=0.002, \eta_{p}^{2}=0.518$, which disappeared at the long SOA $(-7 \mathrm{~ms}), F(1,13)=0.158$, $p=697, \eta_{p}^{2}=0.012$. Therefore, the new long-SOA condition seemed to reduce the response-related interference at the long SOA. Notably, as in Experiment 1, the overall delay of RT2 at the short SOA relative to the long SOA-the PRP effectwas more prominent in the Go trials $(685 \mathrm{~ms})$ than in the NoGo trials $(610 \mathrm{~ms})$, indicating that when Task 1 requires a motor response, the PRP effect is even greater, supporting the contribution of the response-related interference in the typical PRP effect involving Task-1 motor response.

The Task-2 difficulty manipulation lengthened RT2 in the difficult condition $(834 \mathrm{~ms})$ than in the easy condition $(572$ $\mathrm{ms})$ by $262 \mathrm{~ms}$ at the long SOA, $F(1,13)=57.616, p<0.001$, $\eta_{p}^{2}=0.816$, which was similar to the corresponding effect in Experiment $1(268 \mathrm{~ms}), F(1,38)=0.019, p=0.890$, $\eta_{p}^{2}=0.001$. Again, the primary focus of the current study is whether this Task-2 difficulty effect is reduced at the short SOA due to Task-1 motor response and whether there is a three-way interaction across the three experimental factors (the two-way interaction between Task-1 motor requirement and Task-2 difficulty appears at the short SOA but not at the 
long SOA). Looking at the short SOA only, as in Experiment 1 , there was a significant reduction of Task-2 difficulty effect due to Task-1 motor response, $F(1,13)=9.199, p=0.010$, $\eta_{p}^{2}=0.414$ : Task-2 difficulty effect was $167 \mathrm{~ms}$ in the Go trials while it was $286 \mathrm{~ms}$ in the NoGo trials (119 ms reduction), indicating absorption of the Task-2 difficulty effect by the response-related bottleneck, consistent with the motorbottleneck hypothesis. Somewhat unexpectedly, looking at the long SOA only, there was still a significant reduction of the difficulty effect in the Go trials $(233 \mathrm{~ms})$ than in the NoGo trials (291 ms) $-58 \mathrm{~ms}$ reduction, suggesting a residual response-related interference even in the new longSOA condition in Experiment 2. Finally, there was a marginally significant three-way interaction across Task-1 motor requirement, Task-2 difficulty, and SOA, $F(1,13)=2.782$, $p=0.119, \eta_{p}^{2}=0.176$ (see lower panels in Fig. 5). Notably, consistent with the motor-bottleneck hypothesis, in the NoGo trials, the difficulty effect was consistent across SOAs (286 ms at the short SOA vs. $291 \mathrm{~ms}$ at the long SOA), $F(1,13)=0.057, p=0.816, \eta_{p}^{2}=0.004$; however, in the Go trials, the difficulty effect was significantly reduced at the short SOA than at the long SOA $(167 \mathrm{~ms}$ at the short SOA vs. $233 \mathrm{~ms}$ at the long SOA), $F(1,13)=9.686, p=0.008$, $\eta_{p}^{2}=0.427$. Such a prominent reduction in the Task- 2 difficulty effect due to the Task-1 motor response at the short SOA relative to the long SOA is the most distinguishable prediction of the motor-bottleneck hypothesis.

\section{ACC analysis}

ACC1 was significantly lower at the short SOA than at the long SOA $(94 \%$ vs. 97\%), $F(1,13)=28.704, p<0.001$, $\eta_{p}^{2}=0.688$. Participants performed significantly better when Task 2 was difficult (96\%) than when it was easy (94\%), $F(1,13)=7.126, p=0.019, \eta_{p}^{2}=0.354$. Such an effect of Task-2 difficulty on ACC1 was even greater at the short SOA $(-4 \%)$ than at the long SOA $(0 \%), F(1,13)=4.735$, $p=0.049, \eta_{p}^{2}=0.267$. There was a significant three-way interaction across Task- 2 difficulty, SOA, and Task-1 motor requirement, suggesting that the greater effect of Task-2 difficulty on ACC1 at the short SOA than at the long SOA was even greater in the Go trials $(-3 \%)$ than in the NoGo trials $(-1 \%), F(1,13)=5.981, p=0.029, \eta_{p}^{2}=0.315$.

ACC2 was significantly lower at the short SOA than at the long SOA $(92 \%$ vs. $96 \%), F(1,13)=32.250, p<0.001$, $\eta_{p}^{2}=0.713$. Task-2 difficulty manipulation showed the expected effect on ACC2: $96 \%$ vs. $92 \%$ in the easy and difficult conditions, $F(1,13)=6.765, p<0.022, \eta_{p}^{2}=0.342$. There was a significant interaction between Task-2 difficulty and SOA, $F(1,13)=26.664, p<0.001, \eta_{p}^{2}=0.672$, suggesting that the difficulty effect was smaller at the short SOA (1\%) than at the long SOA (7\%). However, Task-1 motor requirement did not show any significant main or interaction effects on ACC2 ( $p s>0.05)$.

\section{LRP analysis}

LRP was calculated and analyzed in the same manner as in Experiment 1. The only significant effect on S2-LRP onset was the main effect of SOA $(p=0.019)$ : the onset of the S2-LRP was significantly delayed at the short SOA (1019 ms) relative to the long SOA (367 ms), indicating a lengthening of the premotoric duration of Task 2 as task overlap increased, consistent with the response-selection bottleneck hypothesis. No other effects or interactions on S2-LPR were significant $(p>0.05)$. Notably, as in Experiment 1 , unlike what the response-monitoring hypothesis predicted (see also Table 1), there was not a significant main effect of Task-1 motor requirement on S2-LRP onset at the short SOA.

Regarding LRP-R2 onset, the Task-2 difficulty manipulation showed a marginally significant trend on LRP-R2 onset ( $p=0.081)$ : LRP-R2 occurred earlier in the easy condition $(-132 \mathrm{~ms})$ than in the difficult condition $(-108 \mathrm{~ms})$. However, unlike what the motor-bottleneck hypothesis predicted-a selective lengthening of LRP-R2 when Task 1 requires a motor response in the easy condition of Task 2 at the short SOA - there was no statistically significant evidence to support this prediction.

\section{Discussion}

Relative to Experiment 1, the new long-SOA condition in Experiment 2 (1200 ms rather than $900 \mathrm{~ms})$ yielded a greater PRP effect combined with a smaller effect of Task-1 motor requirement at the long SOA. Most notably, as in Experiment 1 and consistent with the motor-bottleneck hypothesis, there was a significant reduction in the Task-2 difficulty effect when Task 1 required motor response, which was selectively prominent at the short SOA; this pattern was supported by a significant two-way interaction between Task-1 motor requirement and Task-2 difficulty on RT2 at the short SOA as well as a marginally significant three-way interaction. In addition, as in Experiment 1 and again consistent with the motor-bottleneck hypothesis, the S2-LRP was consistent regardless of the Task-1 motor requirement. Such a result is difficult to reconcile with the response-monitoring hypotheses, which predicted a significant main effect of Task-1 motor requirement on the S2-LRP. However, one of the predictions of the motor-bottleneck hypothesis-selective lengthening of LRP-R2 in the easy condition of Task 2 due to Task-1 motor response-was not supported.

One virtue of the standard PRP paradigm, compared to paradigms in which single-task trials and dual-task trials are run in separate blocks, is that the pre-trial preparatory state 
is identical for long SOAs and short SOAs. Nevertheless, it is possible for participants to become increasingly more prepared for Task 2 at long SOAs, once Task 1 has been responded to. In addition, the temporal onset of the Task-2 stimulus is potentially more predictable at long SOAs. This extra preparation would be even more likely in Experiment 2, which employed an even longer long-SOA (1200 ms) than did Experiment 1 (long SOA of $900 \mathrm{~ms}$ ).

Could this extra preparation at long SOAs explain the key interactions on RT2 in the present experiment? The extrapreparation account is consistent with the relatively short RT2 in the long-SOA condition of Experiment 2 than in Experiment 1 (703 ms vs. $875 \mathrm{~ms}$ ), though that RT difference might also reflect a reduced likelihood of encountering a bottleneck. According to Müller-Gethmann, Ulrich, and Rinkenauer (2003), increased preparation might very well facilitate the pre-motoric stages of Task 2. Importantly, however, the increased preparedness for $\mathrm{S} 2$ in the long-SOA condition should reduce the difficulty effect. Yet, that is the exact opposite of what we found (i.e., the difficulty effect was greater at the long SOA than the short SOA). Therefore, although extra preparation at long SOAs is a strong possibility, it cannot explain the observed interaction between SOA and Task-2 difficulty.

\section{General discussion}

Regarding the cause of dual-task interference, researchers traditionally have considered response selection as the sole source, or at least the primary source, while ignoring possible contributions from motor responses (e.g., Pashler, 1984, 1994; Tombu \& Jolicœur, 2003). However, we observed a greater delay in RT2 when Task 1 required a motor response than when it did not (by $86 \mathrm{~ms}$ in Experiment 1 and by $31 \mathrm{~ms}$ in Experiment 2). This delay was even more prominent at the short SOA: a 110-ms delay in Experiment 1 and 69-ms delay in Experiment 2. We observed such a delay of RT2 due to the Task-1 motor response even though our sample was young and our responses-button presses-were very simple, familiar, fast, and ballistic. The present results add to the evidence that dual-task costs are not due exclusively to response-selection interference. Instead, motoric processes also contribute to dual-task costs as some previous researchers have suggested (Bratzke et al., 2008; De Jong, 1993; Keele, 1973; Ulrich et al., 2006).

The present data not only suggest response-related interference but also shed light on the nature of that interference. Two competing views have been investigated. The motor-bottleneck hypothesis asserts that the Task-1 motor response temporarily delays the motor stage of Task 2 (Keele, 1973); in contrast, the response-monitoring hypothesis asserts that Task-1 motor response is accompanied by a resource-demanding monitoring process that further delays the response-selection stage of Task 2 (Welford, 1952). Previous studies reported inconsistent conclusions regarding the two hypotheses. Behavioral data obtained with a Task-1 motor-response manipulation supported the motor-bottleneck hypothesis (Bratzke et al., 2008, 2009), while electrophysiological data with a focus on residual PRP effect supported the response-monitoring hypothesis (Jentzsch et al., 2007). The aim of the present study was to test the two hypotheses by designing a traditional PRP experiment for which the two hypotheses predict different behavioral and electrophysiological results. Specifically, we manipulated the Task-1 motor requirement, Task-2 responseselection difficulty, and SOA in two experiments.

According to the motor-bottleneck hypothesis, the Task-2 difficulty effect can be absorbed into the response-related bottleneck that occurs after the response-selection stage but before the motor stage of Task 2. At the short SOA in both experiments, we observed such a reduction in the Task-2 difficulty effect when Task 1 required a motor response. The response-monitoring hypothesis, however, does not allow such a reduction because it asserts that the response-related bottleneck occurs before the response-selection stage of Task 2. Moreover, although the response-monitoring hypothesis predicted a significant lengthening of the premotoric duration of Task 2 when Task-1 required a motor response, this effect was not statistically significant; furthermore, in Experiment 1 , the numerical trend also behaved in the opposite direction to what was expected by the hypothesis. However, one of the predictions of the motor-bottleneck hypothesis, regarding the lengthening of the LRP-R2 for the easy condition on Go trials, was not supported. We should note that the LRP results, which were not predicted correctly by either hypothesis, might reflect a mistaken assumption about when the LRP (reflecting motor preparation) begins. For example, we assumed that motor preparation begins following selection of the Task-1 response, but it might instead need to wait until the motor refractory period has ended.

In any case, the data are, in general, much more consistent with the motor-bottleneck hypothesis, which asserts that the nature of the response-related bottleneck is motoric: The Task-1 motor stage temporarily delays the motor stage of Task 2 . Therefore, the widely accepted explanation of the dual-task interference in terms of a pure response-selection bottleneck, as depicted in Fig. 1 (Pashler, 1984, 1994), may need to be modified by adding an additional period of slack, which is smaller than the cognitive slack, between the response-selection stage and motor stage of Task 2. 


\section{But cannot we move limbs simultaneously, without a delay?}

Response-related interference could play an important role of protecting the organism from making two conflicting responses. For example, when a person wants to cut vegetables with a knife and also check a watch on the wrist, the response-related interference may serve to space out the two possibly conflicting motor responses. However, as we know from watching people dancing at a party, people appear to move their limbs simultaneously and smoothly. These anecdotal observations seemingly go against the notion of response-related interference in dual- or multitasking, so why is response-related interference observed in a laboratory? There are a few viable explanations. First, the duration of the delay due to response-related interference is somewhat short-in this study, it was less than $100 \mathrm{~ms}$. Such a short delay may not be obvious to the performers or watchers. In addition, extensive practice with dual-tasking may allow the performers, especially experts, to eventually bypass the motoric bottleneck. For example, they might be able to chunk multiple responses together and jointly implement them as a single motor process (for evidence of bypassing the central bottleneck with practice, or at least shortening it dramatically, see Hazeltine, Ruthruff, \& Remington, 2006; Maquestiaux, Laguë-Beauvais, Ruthruff, \& Bherer, 2008; Ruthruff, Van Selst, Johnston, \& Remington, 2006; note, however, that Ruthruff et al. did not find efficient dualtasking between two tasks with manual responses, as in the present study).

\section{Implications for PRP studies using locus-of-slack logic}

Numerous studies have utilized locus-of-slack logic (McCann \& Johnston, 1992; Pashler \& Johnston, 1989) to determine whether a certain mental process is "automatic" in the sense that it can operate without central attentional resources. Locus-of-slack logic requires manipulating not only the SOA but also the difficulty of the target mental process embedded in Task 2. For example, Jung, Ruthruff, Tybur, Gaspelin, and Miller (2012) varied the difficulty of an attractiveness judgment (Task 2) by presenting faces that were either close to or far from the dividing line between attractive and unattractive. If the difficulty effect interacts underadditively with SOA, then such results indicate that the target mental process has occurred prior to the response selection, especially during the cognitive slack where the attentional resources are not available, and therefore the target process is automatic; but if it interacts additively with SOA, then it does require the resources (i.e., non-automatic, as reported by Jung et al., 2012).
The present findings have implications for PRP studies using the locus-of-slack logic. In those studies, participants are typically required to respond to both tasks. Therefore, based on the present findings, Task- 2 performance might be limited by both a response-selection bottleneck and a motoric bottleneck. Although researchers have traditionally taken underadditive interactions to indicate the automaticity of the target mental process in Task 2 , the present study offers an alternative explanation. The target process might have occurred after the response-selection bottleneck (i.e., only after central attentional resources became available), but the difficulty manipulation on the target process was absorbed into the slack created by the motoric bottleneck.

As a remedy for this issue, researchers who utilize the locus-of-slack logic may consider adopting a go-no-go Task 1. If a target process is truly automatic-i.e., does not require the central attentional resources responsible for the response-selection bottleneck-then the underadditivity between difficulty and SOA should be observed for NoGo trials as well as Go trials. Or researchers could adopt a Task 2 with a large (greater than $100 \mathrm{~ms}$ ) difficulty effect so that the effect cannot be completely absorbed into the relatively short motoric-bottleneck delay. If underadditivity is found using locus-of-slack logic, converging approaches are needed to confirm the findings.

We should also note that the present study does not pose a problem for locus-of-slack logic for cases in which additivity was found. However, it does raise a further problem: true additivity should be very difficult to obtain if there really is a late motoric bottleneck. One possibility is that researchers could have ignored a trend towards underadditivity. For example, in two experiments, Jung, White, and Powanda (2019) tested the automaticity of gender categorization using locus-of-slack logic. In each experiment, they did not observe strong underadditivity. However, when they combined the data from both experiments, there was a statistically significant trend of underadditivity. That is, due to a relatively low power of an individual experiment, researchers could have ignored an underadditive trend. Another possibility is that when Task 1 involves vocal responses rather than manual responses (e.g., McCann \& Johnston, 1992; Van Selst, Ruthruff, \& Johnston, 1999), the motoric bottleneck may be diminished or absent, yielding true additivity.

Acknowledgements We thank Jeff Miller for reviewing the manuscript and providing valuable feedback. This study was supported by the Faculty Summer Research Grant Program of the College of Humanities and Social Sciences at Kennesaw State University.

Funding This research was supported by the College of Humanities and Social Sciences Faulty Summer Research Grant Program at Kennesaw State University. 


\section{Compliance with ethical standards}

Conflict of interest The authors declare that they have no conflict of interest.

Ethical approval All procedures performed in studies involving human participants were in accordance with the ethical standards of the institutional research committee (Kennesaw State University IRB: 18-056) and with the 1964 Helsinki declaration and its later amendments or comparable ethical standards.

Informed consent Informed consent was obtained from all individual participants included in the study.

Open Access This article is licensed under a Creative Commons Attribution 4.0 International License, which permits use, sharing, adaptation, distribution and reproduction in any medium or format, as long as you give appropriate credit to the original author(s) and the source, provide a link to the Creative Commons licence, and indicate if changes were made. The images or other third party material in this article are included in the article's Creative Commons licence, unless indicated otherwise in a credit line to the material. If material is not included in the article's Creative Commons licence and your intended use is not permitted by statutory regulation or exceeds the permitted use, you will need to obtain permission directly from the copyright holder. To view a copy of this licence, visit http://creativecommons.org/licenses/by/4.0/.

\section{References}

Bratzke, D., Rolke, B., \& Ulrich, R. (2009). The source of executionrelated dual-task interference: Motor bottleneck or response monitoring? Journal of Experimental Psychology: Human Perception and Performance, 35(5), 1413-1426.

Bratzke, D., Ulrich, R., Rolke, B., Schröter, H., Jentzsch, I., \& Leuthold, H. (2008). Motor limitation in dual-task processing with different effectors. The Quarterly Journal of Experimental Psychology, 61(9), 1385-1399.

Coles, M. G. (1989). Modern mind-brain reading: Psychophysiology, physiology, and cognition. Psychophysiology, 26(3), 251-269.

De Jong, R. (1993). Multiple bottlenecks in overlapping task performance. Journal of Experimental Psychology: Human Perception and Performance, 19(5), 965-980.

Delorme, A., \& Makeig, S. (2004). EEGLAB: An open source toolbox for analysis of single-trial EEG dynamics including independent component analysis. Journal of Neuroscience Methods, 134(1), 9-21.

Hazeltine, E., Ruthruff, E., \& Remington, R. W. (2006). The role of input and output modality pairings in dual-task performance: Evidence for content-dependent central interference. Cognitive Psychology, 52(4), 291-345.

Jentzsch, I., Leuthold, H., \& Ulrich, R. (2007). Decomposing sources of response slowing in the PRP paradigm. Journal of Experimental Psychology: Human Perception and Performance, 33(3), 610-626.

Jung, K., Ruthruff, E., Tybur, J., Gaspelin, N., \& Miller, G. (2012). Perception of facial attractiveness requires some attentional resources: Implications for the "automaticity" of psychological adaptations. Evolution and Human Behavior, 33(3), 241-250.

Jung, K., White, K., \& Powanda, S. (2019). Automaticity of gender categorization: A test of the efficiency feature. Social Cognition, $37(2), 122-144$
Karlin, L., \& Kestenbaum, R. (1968). Effects of number of alternatives on the psychological refractory period. Quarterly Journal of Experimental Psychology, 20(2), 167-178.

Keele, S. W. (1973). Attention and human performance. Palisades, CA: Goodyear.

Maquestiaux, F., Laguë-Beauvais, M., Ruthruff, E., \& Bherer, L. (2008). Bypassing the central bottleneck after single-task practice in the psychological refractory period paradigm: Evidence for task automatization and greedy resource recruitment. Memory \& Cognition, 36(7), 1262-1282.

McCann, R. S., \& Johnston, J. C. (1992). Locus of the single-channel bottleneck in dual-task interference. Journal of Experimental Psychology: Human Perception and Performance, 18(2), 471-484.

Miller, J., \& Hackley, S. A. (1992). Electrophysiological evidence for temporal overlap among contingent mental processes. Journal of Experimental Psychology: General, 121(2), 195-209.

Miller, J., Patterson, T., \& Ulrich, R. (1998). Jackknife-based method for measuring LRP onset latency differences. Psychophysiology, 35(1), 99-115.

Miller, J., \& Ulrich, R. (1998). Locus of the effect of the number of alternative responses: Evidence from the lateralized readiness potential. Journal of Experimental Psychology: Human Perception and Performance, 24(4), 1215-1231.

Mordkoff, J. T., \& Gianaros, P. J. (2000). Detecting the onset of the lateralized readiness potential: A comparison of available methods and procedures. Psychophysiology, 37(3), 347-360.

Müller-Gethmann, H., Ulrich, R., \& Rinkenauer, G. (2003). Locus of the effect of temporal preparation: Evidence from the lateralized readiness potential. Psychophysiology, 40(4), 597-611.

Osman, A., \& Moore, C. M. (1993). The locus of dual-task interference: Psychological refractory effects on movement-related brain potentials. Journal of Experimental Psychology: Human Perception and Performance, 19(6), 1292-1312.

Pashler, H. (1984). Processing stages in overlapping tasks: Evidence for a central bottleneck. Journal of Experimental Psychology: Human Perception and Performance, 10(3), 358-377.

Pashler, H. (1994). Dual-task interference in simple tasks: Data and theory. Psychological Bulletin, 116(2), 220-244.

Pashler, H., \& Johnston, J. C. (1989). Chronometric evidence for central postponement in temporally overlapping tasks. The Quarterly Journal of Experimental Psychology, 41-A(1), 19-45.

Praamstra, P., Schmitz, F., Freund, H.-J., \& Schnitzler, A. (1999). Magneto-encephalographic correlates of the lateralized readiness potential. Cognitive Brain Research, 8(2), 77-85.

Ruthruff, E., Johnston, J. C., \& Van Selst, M. V. (2001). Why practice reduces dual-task interference. Journal of Experimental Psychology: Human Perception and Performance, 27(1), 3-21.

Ruthruff, E., Van Selst, M., Johnston, J. C., \& Remington, R. W. (2006). How does practice reduce dual-task interference: Integration, automatization, or just stage-shortening? Psychological Research, 70(2), 125-142.

Sammer, G., Blecker, C., Gebhardt, H., Kirsch, P., Stark, R., \& Vaitl, D. (2005). Acquisition of typical EEG waveforms during fMRI: SSVEP, LRP, and frontal theta. Neuroimage, 24(4), 1012-1024.

Schubert, T. (1999). Processing differences between simple and choice reactions affect bottleneck localization in overlapping tasks. Journal of Experimental Psychology: Human Perception and Performance, 25(2), 408-425.

Schwarzenau, P., Falkenstein, M., Hoormann, J., \& Hohnsbein, J. (1998). A new method for the estimation of the onset of the lateralized readiness potential (LRP). Behavior Research Methods, Instruments, \& Computers, 30(1), 110-117.

Shiffrin, R. M., \& Schneider, W. (1977). Controlled and automatic human information processing: II. Perceptual learning, automatic attending, and a general theory. Psychological Review, 84(2), 127-190. 
Sommer, W., Leuthold, H., \& Schubert, T. (2001). Multiple bottlenecks in information processing? An electrophysiological examination. Psychonomic Bulletin \& Review, 8(1), 81-88.

Strobach, T., \& Schubert, T. (2017). Mechanisms of practice-related reductions of dual-task interference with simple tasks: Data and theory. Advances in Cognitive Psychology, 13(1), 28-41.

Telford, C. W. (1931). The refractory phase of voluntary and associative responses. Journal of Experimental Psychology, 14(1), 1-36.

Tombu, M., \& Jolicœur, P. (2003). A central capacity sharing model of dual-task performance. Journal of Experimental Psychology: Human Perception and Performance, 29(1), 3-18.

Ulrich, R., Fernández, S. R., Jentzsch, I., Rolke, B., Schröter, H., \& Leuthold, H. (2006). Motor limitation in dual-task processing under ballistic movement conditions. Psychological Science, 17(9), 788-793.

Ulrich, R., \& Miller, J. (2001). Using the jackknife-based scoring method for measuring LRP onset effects in factorial designs. Psychophysiology, 38(5), 816-827.

Ulrich, R., \& Miller, J. (2008). Response grouping in psychological refractory period (PRP) paradigm: Models and contamination effects. Cognitive Psychology, 57(2), 75-121.
Van Selst, M., Ruthruff, E., \& Johnston, J. C. (1999). Can practice eliminate the psychological refractory period effect? Journal of Experimental Psychology: Human Perception and Performance, 25(5), 1268-1283.

Vince, M. A. (1948). The intermittency of control movements and the psychological refractory period. British Journal of Psychology, $38(3), 149-157$.

Welford, A. T. (1952). The 'psychological refractory period' and the timing of high-speed performance-A review and a theory. British Journal of Psychology, 43(1), 2-19.

Publisher's Note Springer Nature remains neutral with regard to jurisdictional claims in published maps and institutional affiliations. 